\title{
Role of embryonic stem cell-hydroxyapatite construct with growth proteins for osteogenesis in the repair of bone defects in rabbit model
}

\begin{abstract}
Embryonic stem cells (ESC) represent an attracting cell population for tissue engineering purpose. The objective of this study was to determine whether the addition of bone morphogenetic protein (BMP-2), transforming growth factor- $\beta$ and insulin-like growth factor (IGF-1) to a calcium hydroxyapatite (HA-TCP)-rabbit embryonic stem cell (rESC) construct promoted bone healing in a segmental bone defect in rabbits. A $5 \mathrm{~mm}$ long radial ostectomy was performed unilaterally in thirty-six rabbits divided equally into six groups. Defects were filled with a cell-free HA-TCP bioceramic scaffold only (group B); HA-TCP scaffold seeded with rESC (group C); HA-TCP scaffold seeded with rESC along with BMP-2, TGF- $\beta$ and IGF-1 in groups D, E, and F respectively. An empty defect served as the control group (group A). Radiographically, bone healing was evaluated at 10, 20, 30 and 40days post implantation. Histological qualitative analysis by haematoxylin and eosin (H\&E) staining and scanning electron microscopic (SEM) examination was performed 40 days after implantation. Radiographic and histological observation depicted incipient bone formation and filling the defect was more expeditious and better in ESC group than control and bioceramic groups. However, overall faster and better rate of bone healing was observed in the growth proteins added constructs groups (D, E and F) at day 40 after surgery as compared to other groups could be attributed to the application of growth factors in these groups along with the additive effect of the transplanted embryonic stem cells.
\end{abstract}

Volume 4 Issue 3 - 2018

\author{
Bhagyashree K Bhadane,' Swapan Kumar \\ Maiti, ${ }^{2}$ Divya Mohan, ${ }^{2}$ Sadhan Bag, ${ }^{3}$ Naveen \\ Kumar $^{2}$ \\ 'Department of Veterinary Surgery \& Radiology, College of \\ Veterinary \& Animal Sciences, India \\ ${ }^{2}$ Surgery Division, ICAR-Indian Veterinary Research Institute, \\ India \\ ${ }^{3}$ Division of Physiology and Climatology, ICAR-Indian Veterinary \\ Research Institute, India
}

Correspondence: Bhagyashree K Bhadane, Assistant Professor, Department of Veterinary Surgery \& Radiology, College of Veterinary \& Animal Sciences, Parbhani, Maharashtra, India, Tel +91-9412049169,

Emailswapanivri@gmail.com,maiti_62@rediffmail.com

Received: March 08, 2018 | Published: August 16, 2018

Keywords: embryonic stem cell, bone morphogenetic protein, insulin-like growth factor, transforming growth factor, hydroxyapatite bioceramic, rabbit, bone healing

Abbreviations: rESC, Rabbit Embryonic Stem Cells; DPBS, Dulbecco's Phosphate Buffer Saline; DMEM, Dulbecco's Modified Eagles Medium; FBS, Fetal Bovine Serum; HA, Hydroxyapatite; TCP, Tri-Calcium Phosphate; BMP, Bone Morphogenetic Protein; TGF, Transforming Growth Factor; IGF, Insulin-like Growth Protein; MSC, Mesenchymal Stem Cell; SEM, Scanning Electron Microscopy; TEC, Tissue-engineered construct; BFF, Buffalo Fetal Fibroblast; Oct-4, Octamer-4; FFD, Film Focal Distance; kVp, Kilo Voltage Peak; mAs, Milliamperage-Seconds; LIF, Leukemia Inhibiting Factor; H\&E, Haematoxylin and Eosin; HMDS, Hexamethyldisilazine; PMSG, Pregnant Mare Serum Gonadotropin; HCG, Human Chorionic Gonadotropin, ICM, Inner Cell Mass.

\section{Introduction}

Bone is the only tissue in the body able to heal without scarring microscopically. On the other hand fracture healing is a slow process causing long immobilization periods with consequently high costs for fracture healing giving rise to delayed healing, fibrous healing or non-healing with subsequent problems for both, the patient and the orthopaedic surgeon. ${ }^{1,2}$ Fracture healing is a complex phenomenon which involves numerous cells, regulators of cell function and biochemical interactions in the repair process. ${ }^{3-5}$ The healing potential of bone, in a fracture model, is influenced by a variety of biochemical, biomechanical, cellular, hormonal and pathological mechanisms. A continuous occurring state of bone deposition, resorption and remodeling facilitate the healing process. ${ }^{6}$

Biodegradable and bioinert ceramic materials such as hydroxyapatite (HA), tri-calcium phosphate (TCP), aluminumcalcium-phosphate (ALCAP) ceramics provides a scaffold to support the attachment and migration of newly formed bone cells into the osseous defect and also help in the formation of a vascular network through the newly formed bone. ${ }^{7}$ Bioceramic also acts as a carrier to deliver stem cells for osteogenesis. ${ }^{8}$ Specific bone-inductive proteins/ growth factors can induce bone formation and healing in vivo. These morphogens are, therefore, an ideal alternative to autogenous bone grafts. These findings initiating intensive research into bone regeneration orchestrated by putative soluble signals and lead to the discovery and identification of an entirely novel family of protein initiators collectively called the bone morphogenic protein (BMPs), which belong to transforming growth factors- $\beta$ (TGF- $\beta$ ) super family. ${ }^{9,10}$ These growth factors might possess a biological activity that is site and tissue-specific in the induction of bone formation. Different growth factors have been demonstrated to possess osteoinductive and fracture healing properties. ${ }^{11}$

Stem cells have therapeutic potential in the realm of orthopaedic surgery because of their capacity to self-renewal and differentiation into various types of mature cells and tissues, including bone. ${ }^{12}$ Embryonic stem cells (ESCs) are pluripotent cells derived from the inner cell mass of pre-implantation embryos and represent embryonic precursor cells that give rise to any cell type in the embryo. Specifically, it has been shown that ES cells can differentiate into osteogenic cells under selective culture conditions. ${ }^{13}$ These osteogenic cells are capable of in vitro producing bone nodules in three-dimensional scaffolds. ${ }^{14}$

The potentials for using osteogenic stem cells in orthopaedic surgeries for bone and cartilage defect healing is immense and is likely to further expand significantly in future. These approaches have included the implantation of osteoconductive extracellular matrix scaffold and the implantation of bone morphogenic proteins in various matrices. ${ }^{15}$ The most novel approach for this purpose is to combine a scaffold-matrix, living cells and biologically active molecules 
or factors together to form a tissue engineered construct (TEC) to promote the repair and regeneration of bony tissue. ${ }^{16,17}$

However, very fewer approaches have been attempted in direct implantation of pluripotent embryonic stem cells that have been loaded onto a porous ceramics into the bony defects. So, the present study, was conducted to investigate the potential of cultured allogenic embryonic stem cells seeded in a calcium hydroxyapatite (HA/TCP) bioscaffold to generate new bone formation in a segmental bone defect with or without growth factors BMP-2, TGF- $\beta 1$ and IGF-1 in a rabbit model.

\section{Materials and methods}

\section{Experimental design}

The study was conducted on 36 male New Zealand White adult rabbits $(2.58+0.48 \mathrm{~kg}, 6-10 \mathrm{months}$ old $)$ divided into six equal groups (each $\mathrm{n}=6$ ). They were kept in individual cages, fed a standard diet and allowed free mobilization during the study. The groups were compared as follows: The radial 5-mm segmental diaphyseal defect was filled with cell-free bioceramic (HA-TCP) only (group B); rESC+bioceramic (group C); rESC+bioceramic+BMP-2 (group D); rESC+bioceramic+TGF- $\beta 1$ (group E); $r E S C+$ bioceramic+IGF-1 (group F). An empty defect served as the control group (group A). Animals were treated in compliance with the guiding principles in the "Care and use of laboratory animals" policy of the author's institution. Preoperatively hematology was performed to ensure the absence of systemic diseases. The experimental animals had no signs of bone or joint disease on the relevant limbs, as assessed by clinical examination and preoperative radiographs.

\section{Bioceramic}

A biphasic composite bioceramic (calcium hydroxyapatite and tricalcium phosphate) namely HA-TCP with elements in the following percentage: calcium hydroxyapatite- $65 \%$ and tricalcium phosphate $35 \%$, and porosity of $200-450 \mu \mathrm{m}$ was used as sterile ceramic granules.

\section{Isolation of rabbit embryonic stem cells}

Embryo recovery and collection: Each female rabbit was injected with 50-75 IU of PMSG intramuscularly in $1 \mathrm{ml}$ of normal saline. After $72 \mathrm{~h}$ of the first injection, female rabbits were injected with HCG 75 IU intravenously in the ear vein. Female rabbits were mated naturally to two bucks by shifting the does into the male cages following HCG treatment. Collection of embryos was done by flushing the uterine horns and oviducts of rabbits 84-96h after mating. Embryos were collected at blastocyst stage by flushing the uterine horn with modified phosphate buffered saline (Figure 1A \& 1B).

Embryo searching: Flushing fluid was collected in sterile Petri dish in laminar flow. Searching of embryos was done immediately under a maximum of $60 \mathrm{x}$ magnification. A sterile pipette was used to clear the debris attached to the embryos, which were washed again at least for two to three times in fresh flushing fluid to remove uterine content.

Derivation of ES Cells: The embryos collected were seeded on buffalo fetal fibroblast feeder layers. In this technique whole embryo, with its zona pellucida were placed on a mitomycin-C treated feeder layer. Two days after plating, the attachment of the whole embryo to the feeder layer was observed and the medium replaced with fresh medium on a daily basis. The trophoectoderm layer began to expand on the 2-3 day of culturing. The inner cell mass (ICM) like cells formed dome structure. The cells were then incubated at $37^{\circ} \mathrm{C}$ in a humified $5 \%$ carbon dioxide environment in a $\mathrm{CO}_{2}$ incubator. Once the flask achieved confluence, cells were passaged 2-3 times to increase the cell population. After ES cells population was established, it was then confirmed by characterization with different markers by immunocyochemistry.

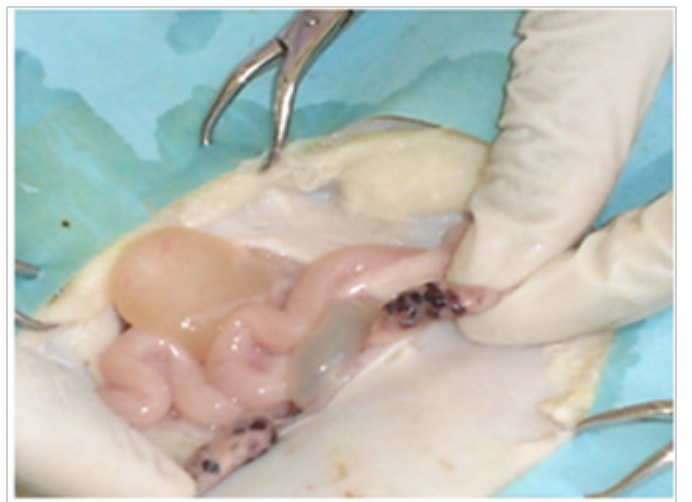

Figure IA Superovulation with large number of Corpus luteum in both ovaries.

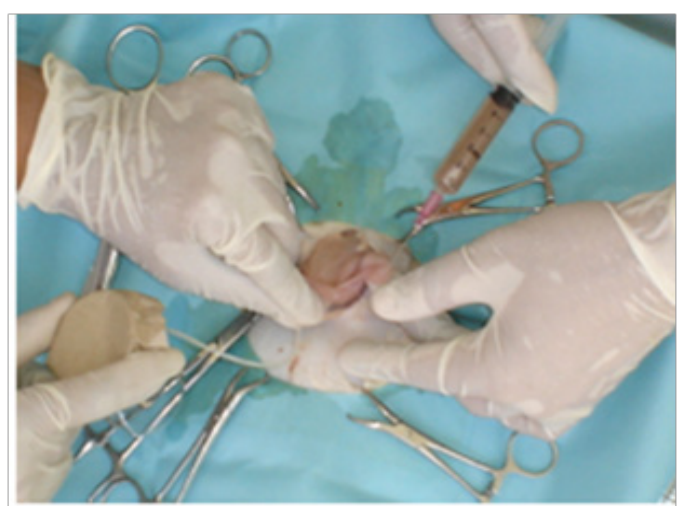

Figure IB Method of flushing of reproductive tract for collection of blastocyst.

\section{Characterization of embryonic stem cells}

Alkaline phosphatase staining: Characterization of stem cell clones with alkaline phosphatase staining was done as follows: Culture medium was removed from the plates, washed three times with $1 \mathrm{x}$ PBS and cells were fixed with $4 \%$ formaldehyde for $20 \mathrm{~min}$. Cells were washed three times in Tris buffer for $10 \mathrm{~min}$ interval between each wash. The last wash was removed, and the staining solution [(Tris buffer: $200 \mu \mathrm{l}$ of a $5 \mathrm{mM} \mathrm{MgCl}$ naphthol AS-MX phosphate, $0.4 \mathrm{mg} / \mathrm{ml}$ : Fast red, $1 \mathrm{mg} / \mathrm{ml}$ )] was added to the cells for 15 to $20 \mathrm{~min}$ and kept it in room temperature for $4 \mathrm{~h}$. Finally washed with 1xPBS and examined under the microscope.

Immuonocytochemistry: The immunocyochemistry was done as per standard method. Briefly, the stem cells were fixed with methanol: acetone $(7: 3)$ for $10 \mathrm{~min}$ at $20^{\circ} \mathrm{C}$ or $4 \%$ formaldehyde in PBS. The cells were washed with PBS and were treated with $3 \% \mathrm{H}_{2} \mathrm{O}_{2}$ in PBS for $10 \mathrm{~min}$ to eliminate internal peroxide and further washed with PBS. Cells on the slide as well as in a culture bottle were then blocked with blocking solution for 30min and then the excess blocking solution was taken out by bloating paper. Primary antibody was added to the cells and again incubated for $180 \mathrm{~min}$ in the moist bath at room temperature. After washing the cells with PBS, a second antibody (FITC conjugated) was added and incubated in the moist bath for overnight at $4{ }^{\circ} \mathrm{C}$ temperature. After incubation, an excess 
of the second antibody was washed with PBS. Di-amino benzidine (DAB) in $3 \% \mathrm{H}_{2} \mathrm{O}_{2}$ was added to the cells and kept in dark for $10 \mathrm{~min}$. The cells were washed gently with the distilled water. The slides were permanently fixed and drop of glycerol was added to the wells of culture plate for observation. FITC treated cells were observed under the fluorescence microscope using a different filter.

\section{Preparation of fibroblast monolayer}

\section{Collection of fetus}

The buffalo uterus containing $2 \mathrm{~cm}$ fetus was aseptically collected from the local slaughterhouse and transported to the laboratory within $1 \mathrm{~h}$. The whole organ was washed thoroughly in sterile NSS supplemented with Gentamycin $(5 \mu \mathrm{g} / \mathrm{ml})$. The fetus was approached after swabbing in ethyl alcohol and then with the help of sterile forceps and scissors, the fetus was taken out and kept in a sterile beaker containing NSS. Finally, skin sample collected from the fetus with the help of sterile forceps and scissors and washed in DPBS.

\section{Isolation of fetal skin fibroblast cells}

Collected fetal skin samples were cut into small pieces and taken in a small beaker containing DPBS and the tissues were disrupted mechanically by a $10 \mathrm{ml}$ disposable sterile pipette attached to a rubber bulb by making the fluid along with tissue in and out of the pipette. By repeated disruption for $20 \mathrm{~min}$, the fluid was checked for isolated individual fibroblast cells. After getting isolated cells, the fluid was collected in a $15 \mathrm{ml}$ centrifuge tube and washed in DPBS and then centrifugation for $5 \mathrm{~min}$, so that the clumped tissue fragments settle at bottom and the supernatant was taken in another $15 \mathrm{ml}$ centrifuge tube and again washed in DPBS for $15 \mathrm{~min}$ by centrifugation and the supernatant was discarded. The remaining fluid containing fibroblast cells was washed in RPMI media added up to $3 \mathrm{ml}$ in the $15 \mathrm{ml}$ centrifuge tube and used for setting monolayer. The cells were plated in $25 \mathrm{~cm}^{2}$ culture flask and 24 well plate in RPMI with $15 \%$ FBS. After the attachment of cells to the surface, the media was replaced by fresh media and attained confluence. Fresh media was replaced every $72 \mathrm{~h}$ for maintaining the culture.

\section{Subculture}

The media was replaced with IX PBS twice and then added trypsin-versene solution and incubated for $5 \mathrm{~min}$. The solution was then replaced by RPMI with 3\% FBS with gentle tapping with fingers The cells became loose and got detached from the plastic surface. The media with the cells were transferred to $25 \mathrm{~cm} 2$ culture flask and 24 well plates. Once confluence, the mitotic growth was blocked by mitomycin-C treatment. Then media containing mitomycin-C was removed and the wells were washed with IX PBS at least 2 times. Further, in the wells, media containing DMEM with $15 \%$ FBS and LIF (Leukemia Inhibitory Factor) was added. This treated monolayer was used for growing the embryonic stem cells.

\section{Surgical procedure}

All the animals were acclimatized for three weeks to approaching and handling prior to surgery. Under general anaesthesia with xylazine hydrochloride $(5 \mathrm{mg} / \mathrm{kg}, \mathrm{IM})$ and ketamine hydrochloride $(50 \mathrm{mg} / \mathrm{kg}$, IM) and strict surgical asepsis, a unilaterally $5 \mathrm{~mm}$ bone defects were created in the radial diaphysis. A $2 \mathrm{~cm}$ medial incision over the radius was given, soft tissue was dissected and the bone exposed by gentle retraction of the muscles. A Hohmann retractor was placed between ulna and radius to protect the ulna. A $5 \mathrm{~mm}$ segmental osteo-periosteal diaphyseal defect was created with an oscillating bone saw that was continuously cooled by irrigation with $0.9 \%$ sterile cold saline solution. The periosteum was removed with the bone and $5 \mathrm{~mm}$ of periosteum was stripped from each side of the remaining radius. The bone gap was irrigated with a sterile physiological saline solution and ceramic granules (or nothing) fitted into the gap as per treatment protocol for different groups. Muscles, fascia and skin were separately closed over the defect with 4-0 resorbable sutures. A wooden splint with bandage was applied to the test bone for one week.

\section{Postoperative care}

Postoperatively, ceftriaxone sodium $(20 \mathrm{mg} / \mathrm{kg}, \mathrm{IM})$ administered intramuscularly for five days. Analgesic meloxicam $(0.2 \mathrm{mg} / \mathrm{kg}, \mathrm{IM})$ was given for the first 3 postoperative days. Water and food were supplied ad libitum. After 40days of the experiment, the animals were sacrificed.

\section{Radiological assessment}

Craniocaudal and mediolateral radiographic view of each ostectomy site was assessed immediately after surgery to monitor the position of the implants, and at 10,20,30 and 40 days postoperatively to evaluate the presence of osseous union at each host bone-implant interface, the presence of osseous callus overlying the implant and the presence of periosteal reaction. The radiographic factors used were $14 \mathrm{mAs} ; 50 \mathrm{kVp}$ and $90 \mathrm{~cm}$ FFD

\section{Scanning electron microscopic assessment}

Scanning electron microscopic (SEM) technique was used for evaluation of new bone formation at the defect site in different treatment groups. At day 40 after implantation, the constructs were explanted after euthanizing the animals. Each specimen consisted of both fracture healing site and adjacent bone. The samples were fixed in $2 \%$ glutaraldehyde phosphate buffer solution for $24-48 \mathrm{~h}$. After fixing in glutaraldehyde, two pieces of washing for 30min each with phosphate buffer saline $(\mathrm{pH}$ 7.4) followed by distilled water was performed. Then the specimens were dehydrated in the serial concentration of ethanol $30-100 \%$ for $30 \mathrm{~min}$ each. Thereafter, the specimens were dried with HMDS for $20 \mathrm{~min}$. Specimens were mounted on aluminium stubs using adhesive silicone tape. After the golden ion sputtering done on Jeol ion sputter Model JFC 1600 at $7-10 \mathrm{~mA}$ and $1-2 \mathrm{kV}$ for $15 \mathrm{~min}$. Finally, the specimens were observed under scanning electron microscope at appropriate acceleration voltage and the magnification range of the unit calibrated for the study of their different surfaces to identify the orientation and distribution of newly formed osseous tissue and distribution or absorption of construct at the bone defect site.

\section{Histopathological assessment}

At day 40 post-implantation, the animals were sacrificed. The test bone sections throughout the length of the defect were collected from the site of the surgical implant and washed thoroughly with the sterile normal saline solution. Fixation was done in $10 \%$ formalin. The bone sections were subjected to decalcification in Goodling and Stewart solution $(15 \mathrm{ml}$ formic acid, $5 \mathrm{ml}$ formalin and $80 \mathrm{ml}$ distilled water). The decalcified bone sections were stained by H\&E as per the standard procedure. The sections were examined using a light microscope under different magnification for cellular reaction, healing process and fate of the grafted implant/ECS construct. 


\section{Results}

\section{Superovulation and quality of embryos}

Superovulation was successfully achieved in 6-10 months old female New Zealand rabbits following injection of PMSG-HCG protocol followed by natural mating, during spring and just before the winter season. A very good quality and good numbers (maximum 25) of embryos were collected by flushing the uterine horns and oviducts of rabbits after 84-96h of mating (Figure 1C \& 1D).
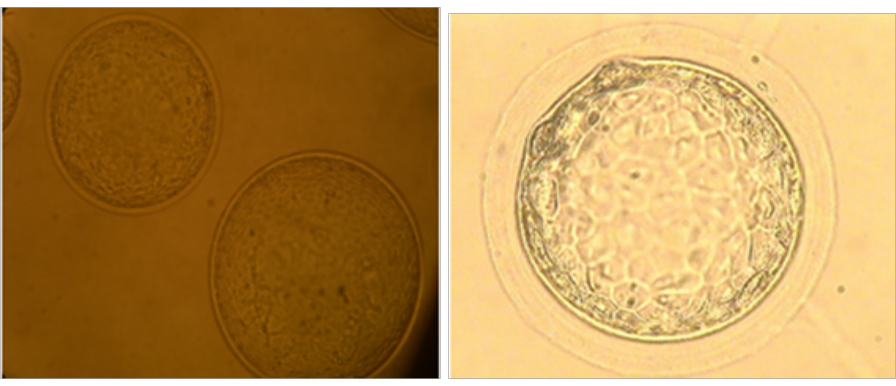

Figure IC \&ID Superior quality of blastocysts immediately after collection.

\section{Embryonic stem cell derivation and propagation}

The medium used for culture and derivation of the stem cells consisted of Knockout Dulbecco's modified Eagle's medium with 5\% FBS and recombinant murine Leukemia Inhibitory Factor (LIF) in 5\% $\mathrm{CO}_{2}$ and $37^{\circ} \mathrm{C}$. This medium was found to be very useful for culture and derivation of rESC. In this study, the whole blastocyst was seeded on the inactivated feeder rather than mechanical isolation of ICM by immunosurgery. The blastocyst stage of embryos those were ready to hatch at the time of collection (Figure $1 \mathrm{E} \& 1 \mathrm{~F}$ ) with bulging of zona pellucida at one point hatched within 4-6h after culture. Some blastocyst took $10-12 \mathrm{~h}$ to hatch. Few blastocysts hatched after 14$16 \mathrm{~h}$ after their culture on a mitomycin-C inactivated BFF feeder. Hatched embryos (Figure 1G \& 1H) showed attachment within 24$48 \mathrm{~h}$ after hatching. Embryos those did not show attachment even after of 2-3days of hatching were discarded and was not used for further study. Approximately 6-8 days after attachment of hatched blastocyst the ICM derived outgrowths were seen. The ICM was allowed to grow on feeder cells for 12-15days before the first transfer of the growing cells to a new feeder plate (Figure 1I). ICM derived outgrowths that were mechanically removed from feeder cells after the perimeter of the outgrowths folded back. The ESC like colonies were mechanically separated from trophoectoderm cells and divided into small clumps by needle, then treated in a drop exposure to $0.05 \%$ trypsin-versine solution for 1 minute at room temperature. To terminate digestion, the culture was rinsed once in PBS, resuspended in fresh culture medium, and mechanically disaggregated into small clumps (Figure 1J). The small ICM cell clumps were seeded on fresh BFF and the ESC- like colonies were seen several days later. The initial outgrowth of the ICM often contained heterogeneous cell types. After growing approximately for 6-8days the colonies were again lifted and passaged further for more separation of cells followed by treatment with $0.05 \%$ trypsin-versine solution for few minutes and was then propagated in clumps of approximately 500 or more cells on fresh BFF (Figure 1K).

\section{Expression of pluripotent markers in cultured embryonic cells}

Embryonic stem cell marker expression was observed in this study. ES cells expressed high levels of alkaline phosphatase (AP) activity as evidenced by its characteristic pink coloration after staining (Figure 1L). Expression of pluripotent markers in cultured embryonic cells were observed by alkaline phosphatase and was further confirmed by Oct-4 in few cases by immunocyochemistry as evidenced by its green fluorescence (Figure $1 \mathrm{M} \& 1 \mathrm{~N}$ ). Colonies those showed positive expression was only used for implantation study in the segmental bone defect in the rabbit model.

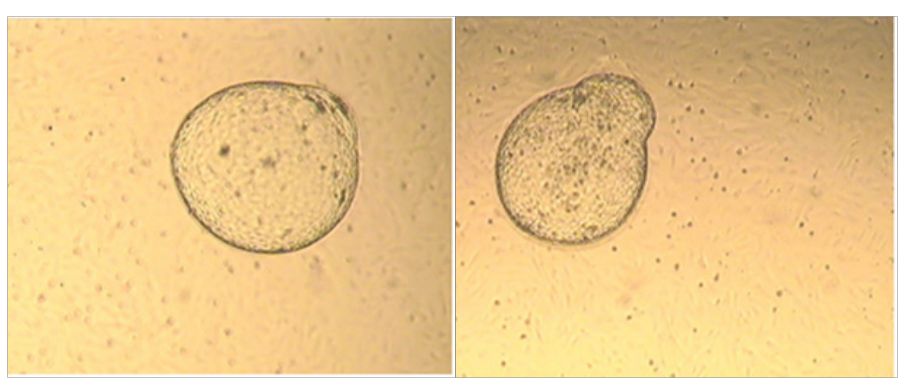

Figure IE \& IF Bulging of blastocyst through zona pellucid.

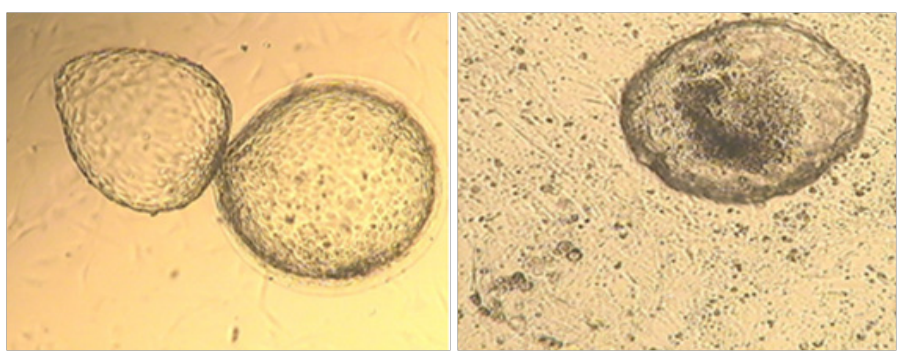

Figure IG \& IH Completely hatched blastocyst.

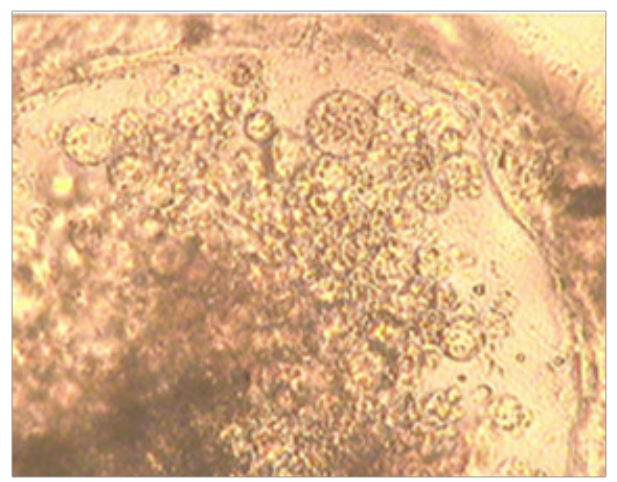

Figure II Growing and expanding inner cell mass (ICM).

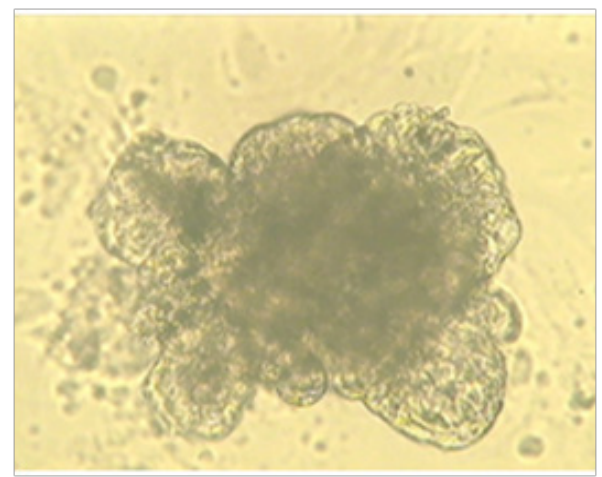

Figure IJ Colony of inner cell mass immediately after mechanical disruption. 


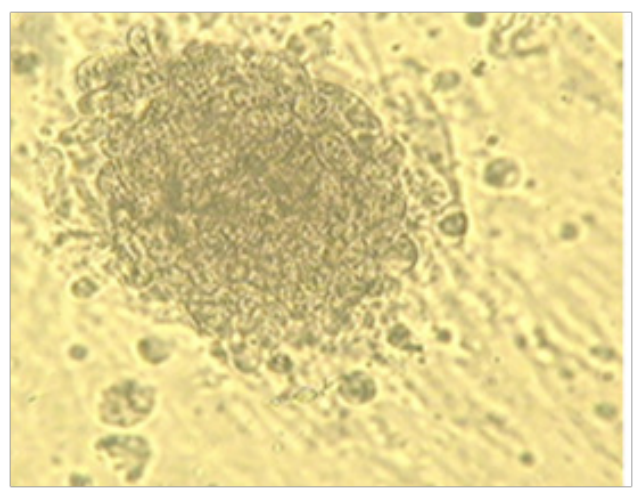

Figure IK ESC colony after passage 2.

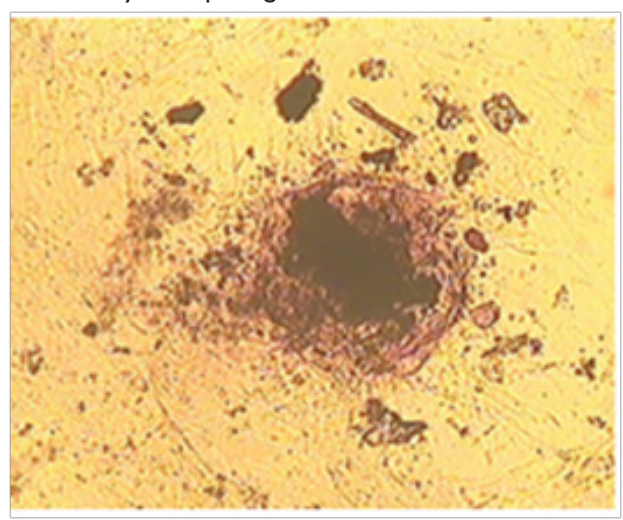

Figure I L Alkaline phosphatase staining of ESC.

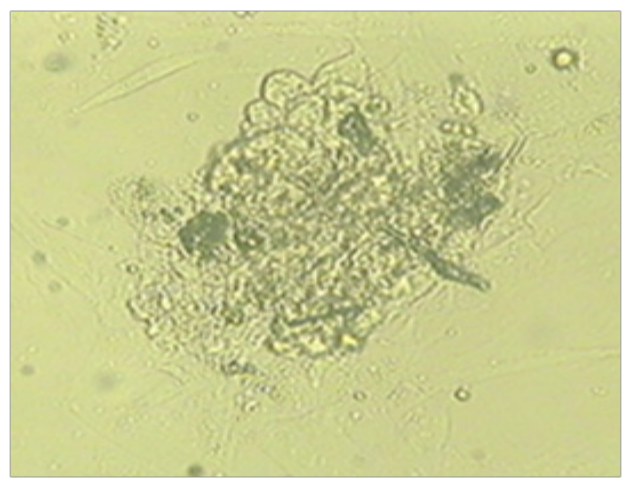

Figure IM ESC colony before staining.

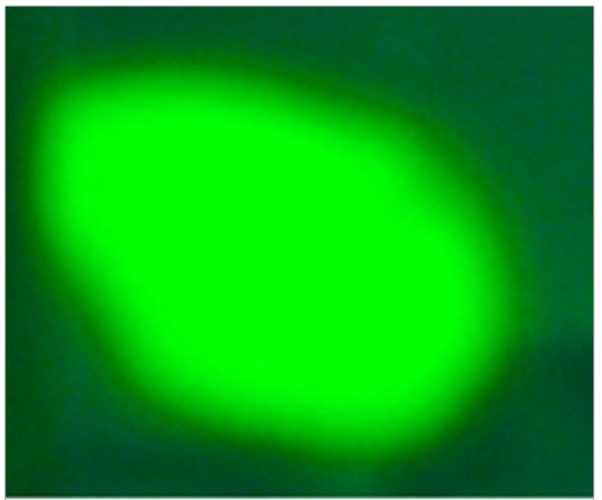

Figure IN Characterization of ESC by Oct 4 (FITC conjugated x I0).

\section{Surgical procedure}

The combination of xylazine and ketamine anaesthesia provided safe and satisfactory anaesthesia for the collection of embryos and during the implantation in rabbits and in none of the animals complications due to anaesthesia were observed. The use of cold sterile normal saline solution at the defect site, while creating the bone defect with the orthopaedic saw, prevented thermal necrosis of the cut edges of host bone. The hydroxyapatite -tricalcium phosphate granules were used in the form of granules and were secured at the defect site by suturing surrounding soft tissues. The process of transplantation of embryonic stem cells and growth factors (BMP2 ; TGF- $\beta$ and IGF-1) at the site of osteotomy was carried out easily without any complication. Suturing of muscles, soft tissues and skin separately helped to secure the implants in position and prevented their migration in all animals. External splint application to the respective forelimb immediately after implant application found very useful to prevent the chances of complete fracture occurrence at the defect site.

\section{Clinical observations}

Clinically, all the animals of different groups behaved identically, surgical wounds healed by the first intention. Inflammatory signs appeared at the fracture site from the first postoperative day in animals of all the groups. Pain showed a further increase in all groups by day 3 to $7 \mathrm{PO}$ and thereafter it showed a downward trend reaching normal by day 15. All the animals of six groups showed signs of lameness in the immediate postoperative period. However, there was no visible difference in lameness between different groups. Weightbearing gradually improved as signs of inflammation subsided within 8-10days.

\section{Radiographic observations}

The radiographic evaluation was done based on mediolateral view as there was overlapping of the radius by ulna in craniocaudal view. Mediolateral views of radiograph of animals of six groups are presented in Figure 2. In control group (A), at day 30, a fibrous density appearance was seen near the defect margins and there was no indication of the filling of the defect with newly formed tissue. At day 40 , radiological observations were more or less the same except that the newly formed osseous tissue at cut ends was more organized and the fractured ends became more smooth and round. In group B, at day 20, HA-TCP bioceramic granules appeared relatively more compact with signs of osteogenesis from the periphery. The periosteal reaction was seen adjacent to the cut ends of radius. On day 40 , the bone defect was partially filled with newly forming bone; however, the implant was still present. There were indications of slowing down of healing process as the edges appeared relatively smooth and newly formed bone was organized. In group $\mathrm{C}$, at day 10 and 20 , the defect site showed increased radiographic density in all animals of this group. The cut ends of the bone looked hazy and irregular with signs of new bone formation on either ends. On day 30, a radiolucent line was present in the middle of the defect showing incomplete bridging of the gap. On day 40, radiographs showed that there was further consolidation of new bone formation and signs of union between the implant and host bone was visible at the proximal end of segmental defect. The implant was looking more compact at this stage indicative of progression of good healing process. In group D, at day 20, the defect site showed increased radiographic density in all animals. The cut ends of the bone looked hazy and irregular with signs of new bone formation on either ends. At day 30 , there was further increase in new bone formation at the defect site. At day 40, all the animals showed 
signs of increased new bone formation and periosteal reaction at defect site as compared to day 30 ; however, still there was radiolucent line visible in between the implant. In group E, at day 20, the signs of new bone formation from both cut ends of radius and radiodensity were increased at the defect site reducing the gap. The defect site was looking slightly denser than the adjacent bone but the marrow cavity was not visible at the site of the defect. At day 30 , the defect site was completely healed and fused with the adjacent host bone in 4 animals. In remaining animals, new bone growth was seen from bone ends and only a small radiolucent line was visible between the implant and cut end of bone at distal fragment. On day 40, the defect site appeared more radiodense with complete union between the implant and bone at proximal end of defect. In group F, at day 10, periosteal reaction was started. At day 20, there was increased radiodensity at the defect site, which shows signs of new bone formation. Cartilaginous density was observed at the defect margins on day 30 . On day 40 the whole defect showed cartilaginous density whereas the defect margins showed bony density and bridging the defect ends.

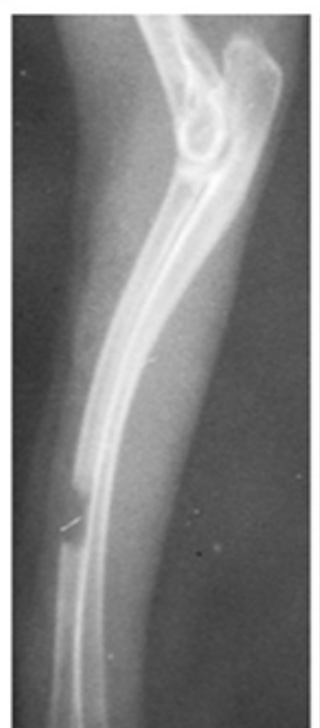

Day 0

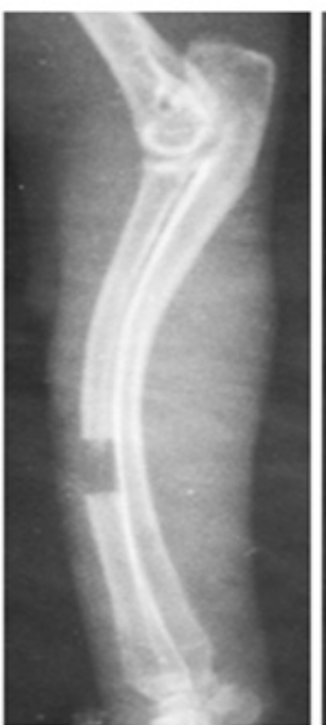

10

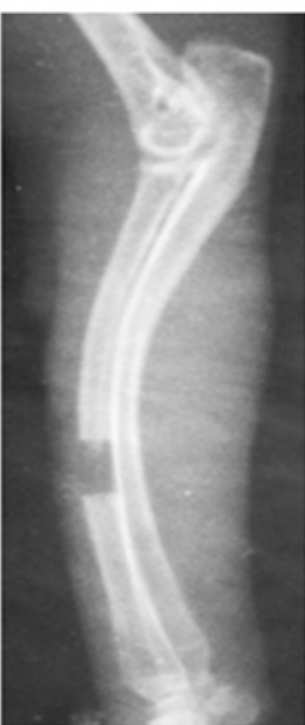

20

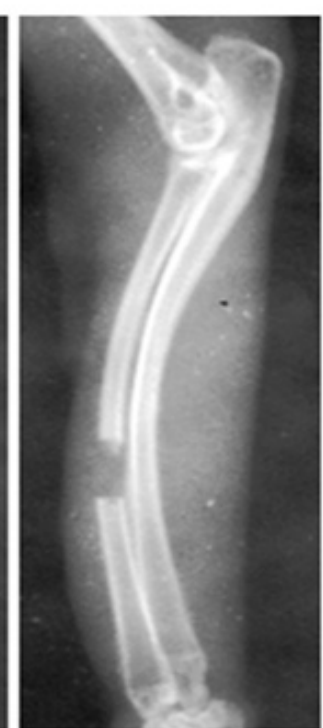

30

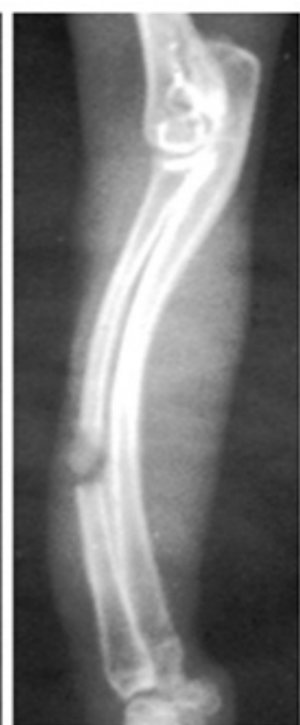

40

Figure 2A Sequential radiographs of gr $\mathrm{A}$.

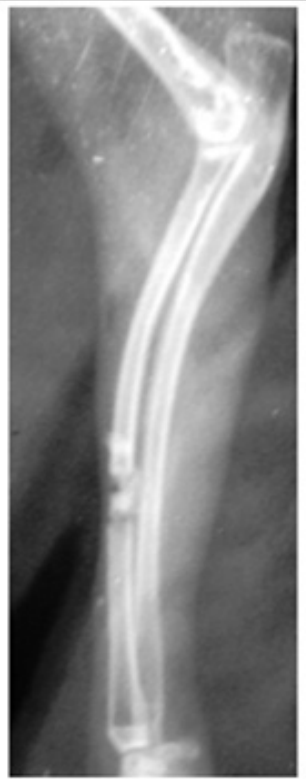

Day

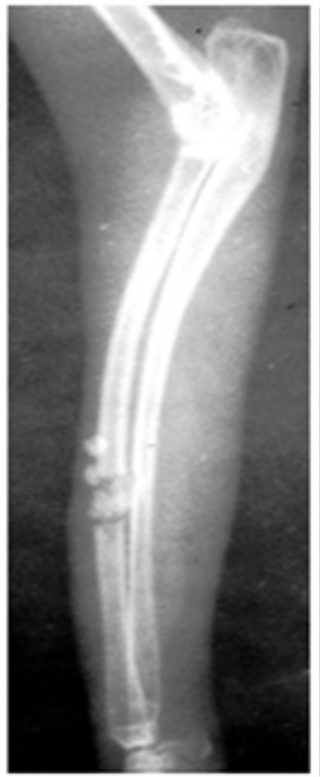

10

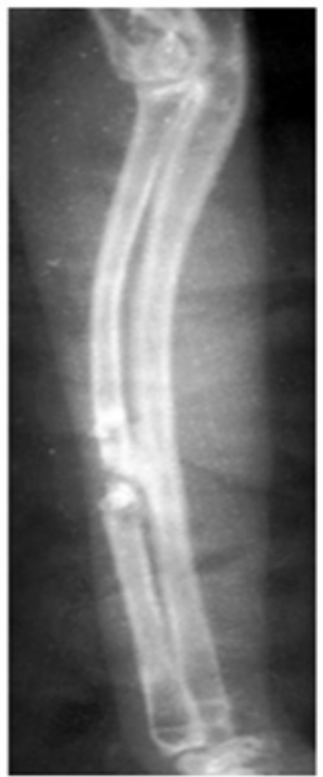

20

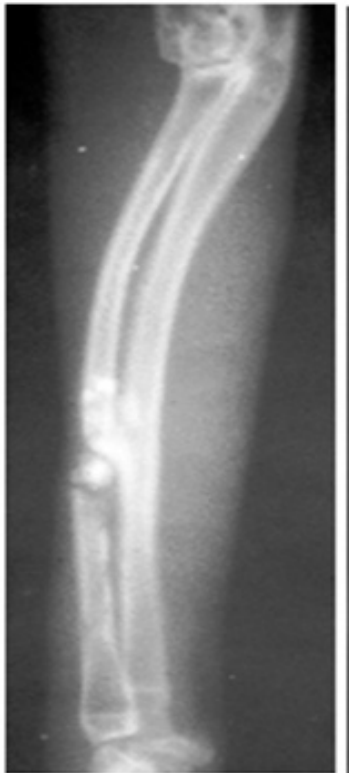

30

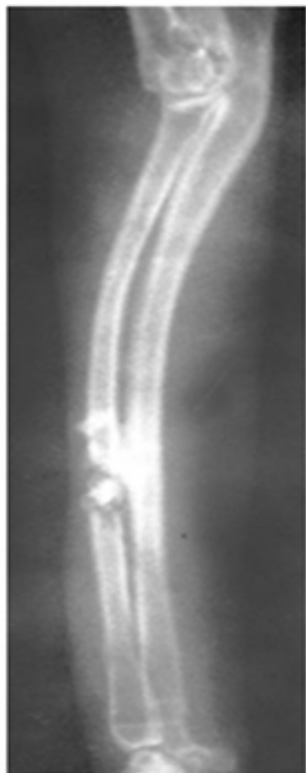

40

Figure 2B Sequential radiographs of gr B. 


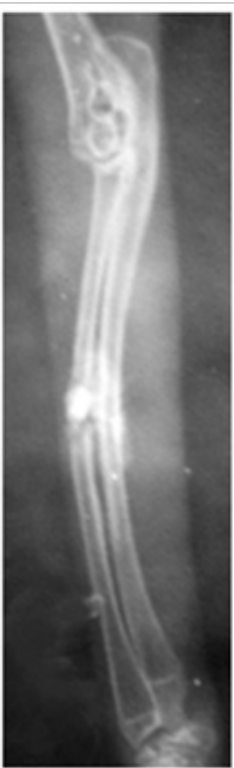

Day

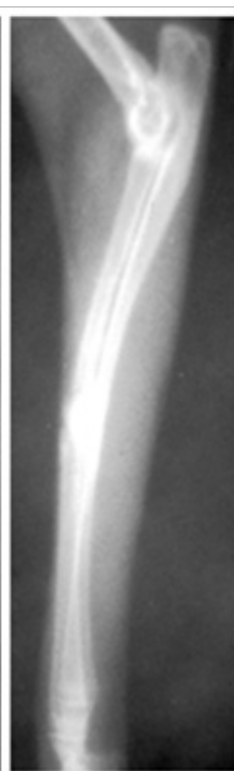

10

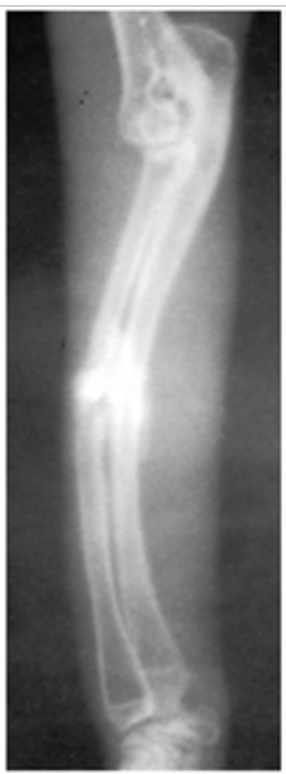

20

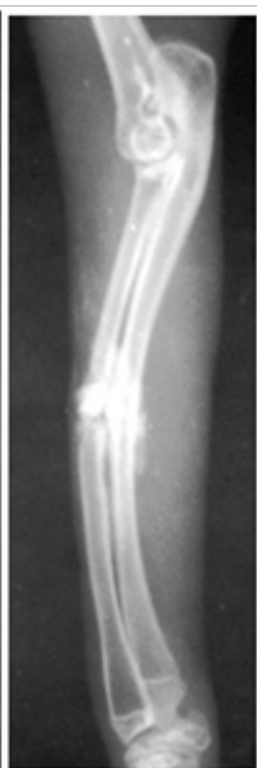

30

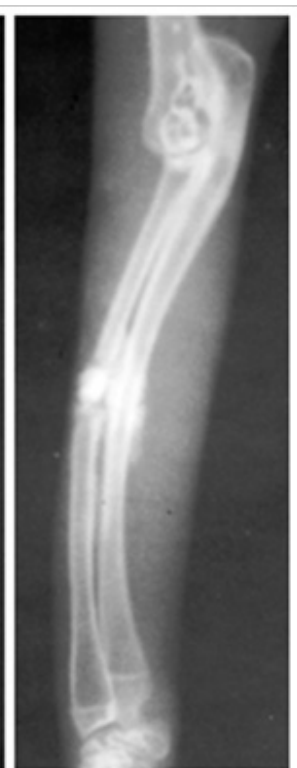

40

Figure 2C Sequential radiographs of gr $\mathrm{C}$.

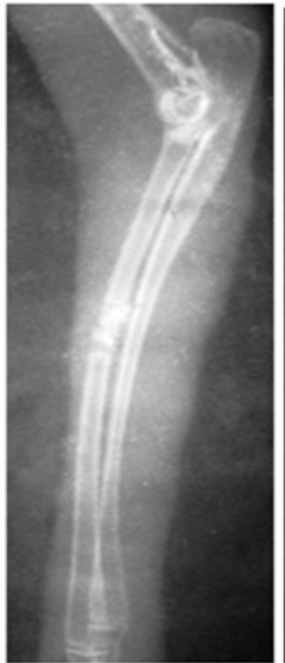

Day

$\mathbf{0}$

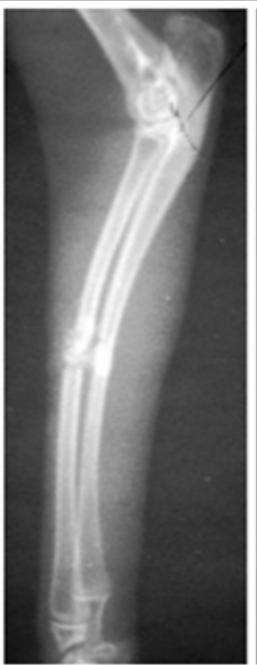

10

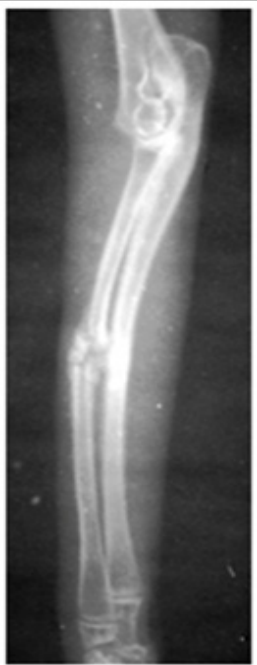

20

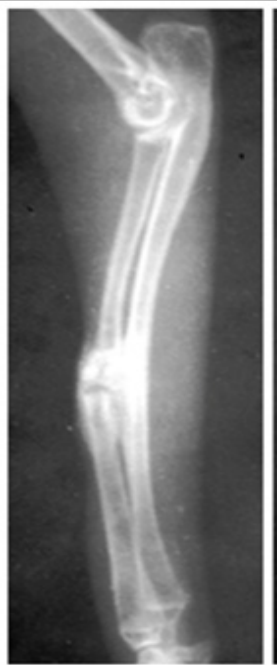

30

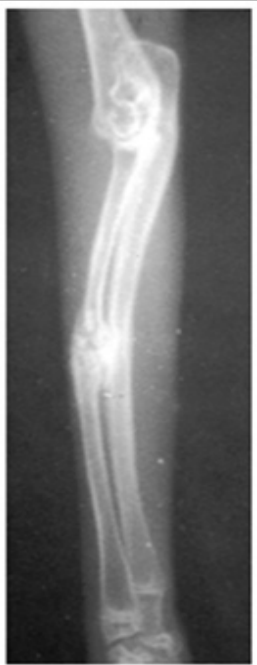

40

Figure 2D Sequential radiographs of gr D.

\section{Histological observations}

Histological sections at bone defect site of different groups at day 40 are presented in Figure 3. In control animals (A), the majority of the defect area was occupied by fibrocartilagenous tissue. However, there was no continuity between fibrous tissue from the proximal and distal fragments. In group B, the defect site depicting penetration of fibrous connective tissue in the ceramic particles and scanty new bone formation adjacent to fibrous connective tissues. There were some signs of resorption of implanted material. In group C, the histological picture at the defect site showed only partial degradation and absorption of HA-TCP particles. There was no infiltration of any inflammatory cells. However, the connective tissue below the periosteum and underneath the implanted construct mass was moderately thick, with signs of osseous cavity at the edges and large number of enlarged chondrocytes. The bone defect was almost repaired with newly formed woven bone. In group $\mathrm{D}$, the thick periosteal connective tissue covering over the defect site which was seen invading in and around the implanted bioceramic, which was entrapped within the interlacing osteoid trabeculae. There was evidence of hypertrophied chondrocytes indicating the bone formation through endochondral ossification. The decalcified bone sections showing formation of marrow elements in the resorption cavities as well as fibrocartilagenous connective tissues at the center of bone defect. In group E, the thick band of fibrous connective tissue was seen invading the implanted material from all sides. The interface of the implant-host bone showed intense 
osteoblastic activity, presence of new blood vessels at the border of the implant and towards the endosteum. The new bone formation was found to be comparatively better than groups B and C. In group $\mathrm{F}$, bone sections revealed the presence of cartilaginous and osseous tissue evidenced by osteoblasts. The osteoid tissue was more prominent forming a woven bone pattern. There was complete filling of the defect area by new bone growth.

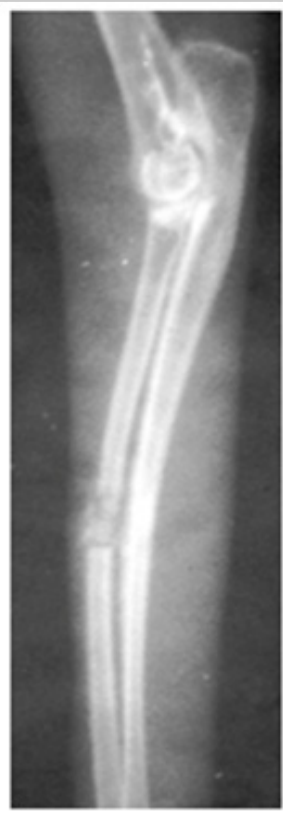

Day

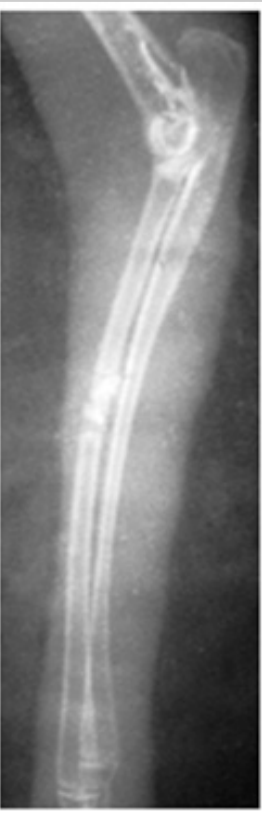

10

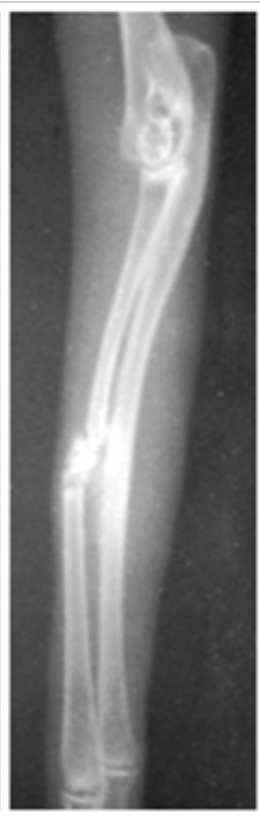

20

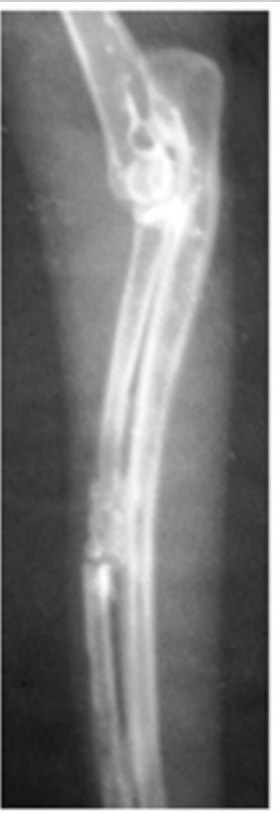

30

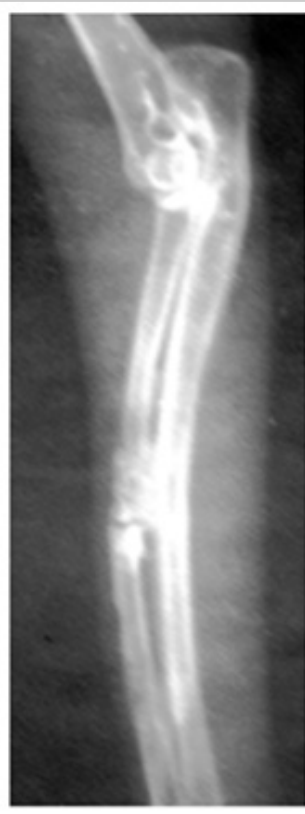

40

Figure 2E Sequential radiographs of gr E.

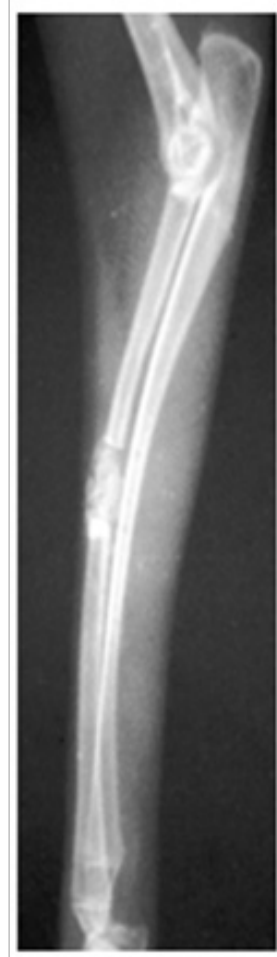

Day

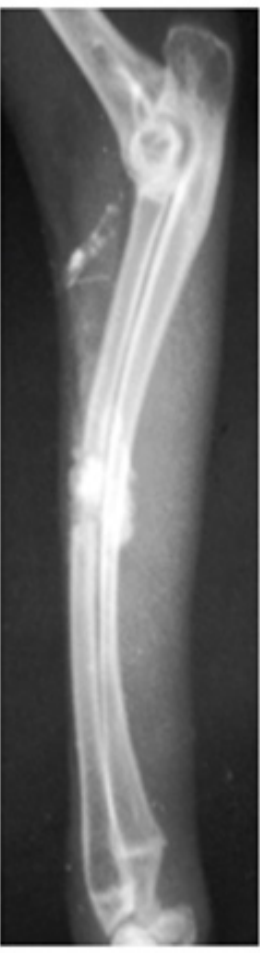

10

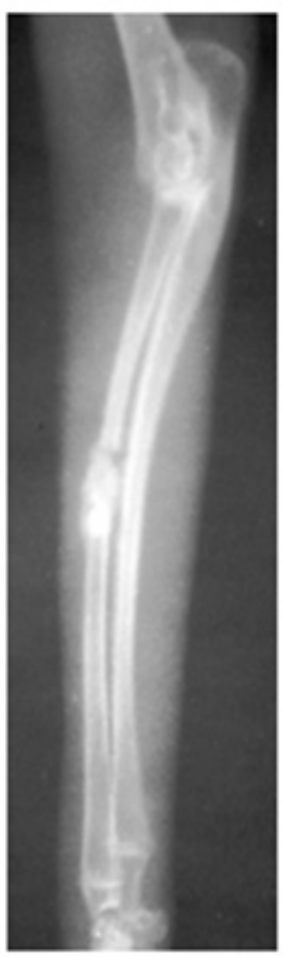

20

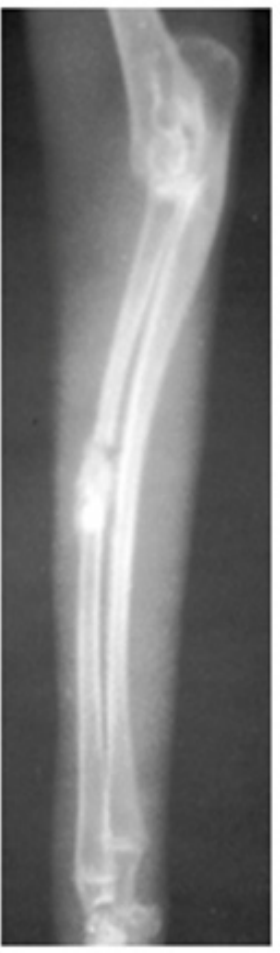

30

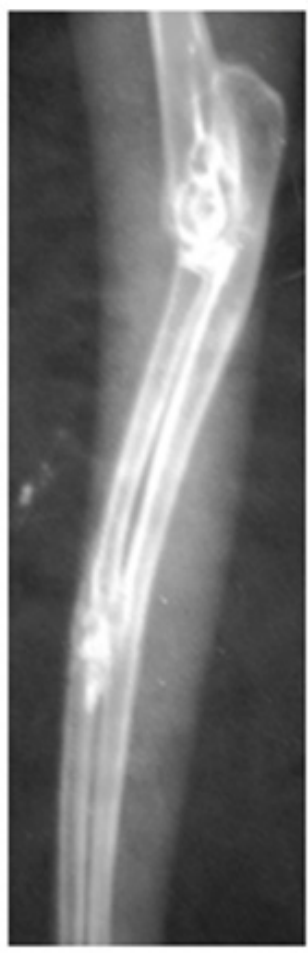

40

Figure 2F Sequential radiographs of gr $\mathrm{F}$. 


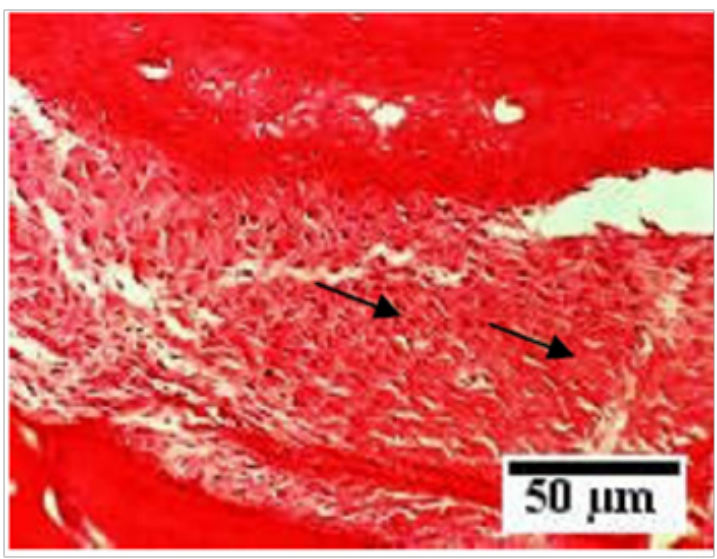

Figure 3A Transverse section of gr A showed fibrocartilagenous tissue $(\rightarrow)$ at the defect site (H\&E, IOOX).

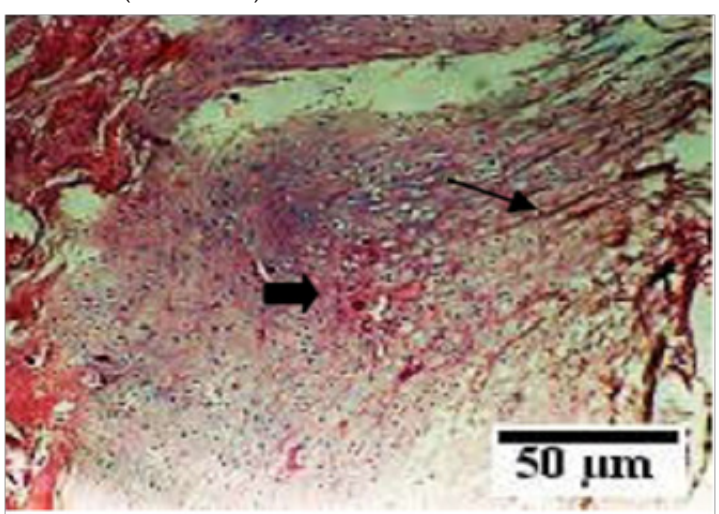

Figure 3B Transverse section of gr B depicted penetration of fibrous connective tissue $(\rightarrow)$ within the ceramic particles and scanty new bone formation $(\rightarrow)$ adjacent to fibrous connective tissue (H\&E, I00X).

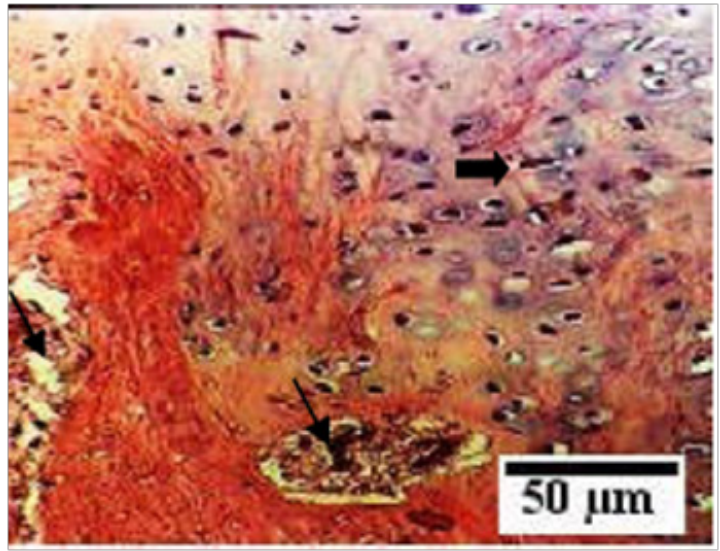

Figure 3CTransverse section of gr $\mathrm{C}$ showed new bone formation with signs of osseous cavity $(\rightarrow)$ at the edges and large number of enlarged chondrocytes $(\rightarrow)$ at the defect site (H\&E, I00X).

\section{Scanning Electron Microscopic (SEM) study}

SEM analyses at bone defect site of different groups at day 40 are presented in Figure 4. In group A, the gap was filled by newly proliferated tissue which was a mixture of cartilaginous and fibrous tissues. The fibro cartilage was present at the centre of the defect. The adjacent periosteal surface of the ulna was also attached with healing site through newly proliferated tissues. However, it was the only fibroblast that invaded the defect. In group B, HA-TCP particles were seen projecting over the surface. These particles were seen surrounded by newly formed bone matrix suggesting that bioceramic provided base for new bone formation, however, it failed to incorporate with newly formed bones. In group $\mathrm{C}$, the healing process of new bone formation was relatively faster as compared to group B. The ceramic particles were seen fused with newly formed bone matrix suggesting their incorporation with newly formed osseous tissue. The unorganized osseous tissue had advanced from the cut edges into the implant material. The area of the implanted site was almost completely occupied by the bone matrix. The extracellular matrix was well organized at many places indicating the maturity of the osseous tissue. In group D, the defect site revealed some irregular area in the centre and at the host-implant junction, there was presence of compact bone suggesting advanced stage of healing. In group E, the SEM picture depicted compact bone formation at the host and implant junction towards the periosteal surface of the bones. At few places, small pits were discernable which could be due to the absorption of the small HA-TCP granules. Further magnification of the implanted site showed well organized trabecular bone embedded in the implanted material which was surrounded by compact bone at places. In group $\mathrm{F}$, scanning micrograph of healing site showed some irregular areas in the centre of the defect and at the junction of the bioceramic construct. At the cut edges in this group, there was presence of compact bone suggesting advance stage of healing.

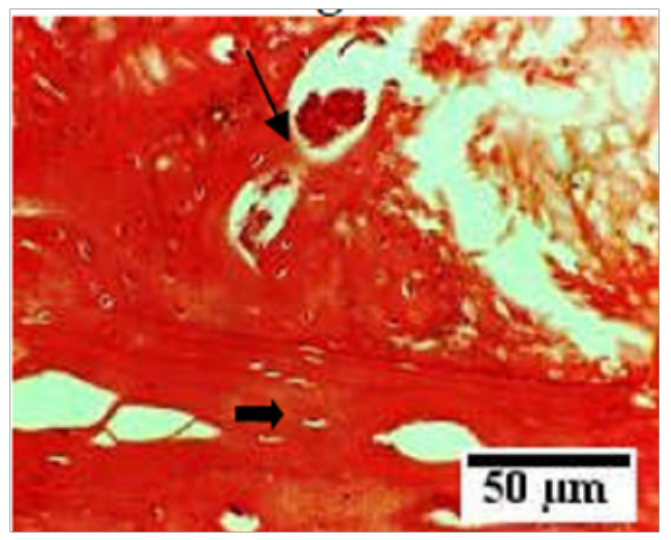

Figure 3D Transverse section of gr $\mathrm{D}$ showed formation of marrow elements $(\rightarrow)$ in the resorption cavities as well as fibrocartilagenous connective tissue $(\rightarrow)$ at the center of defect (H\&E, I00X).

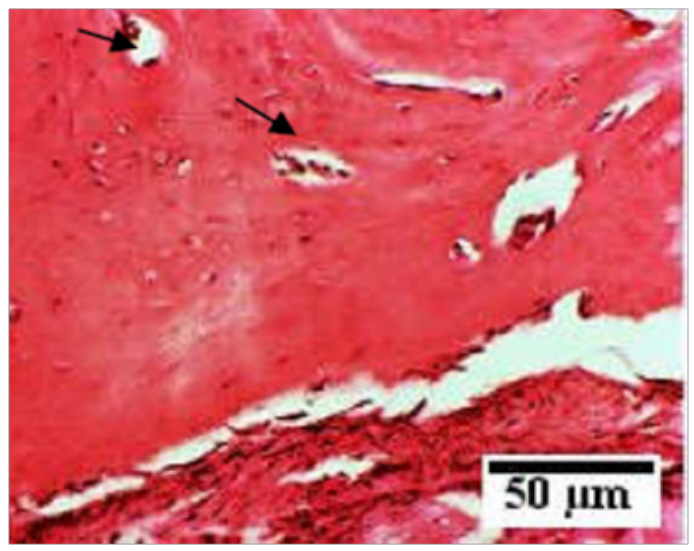

Figure 3E Transverse section of gr E showed formation of new bone and blood vessels $(\rightarrow)$ at the border of the implant and towards the endosteum (H\&E, I00X). 


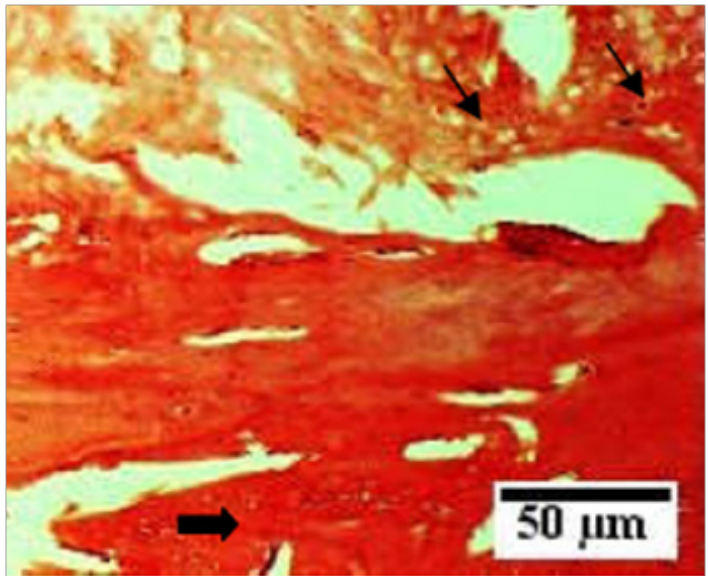

Figure 3F Transverse section of gr $\mathrm{F}$ depicted presence of cartilaginous $(\rightarrow)$ and osseous tissue $(\rightarrow)$ evidenced by osteoblasts at the defect site $(\mathrm{H} \& \mathrm{E}$, I00X).

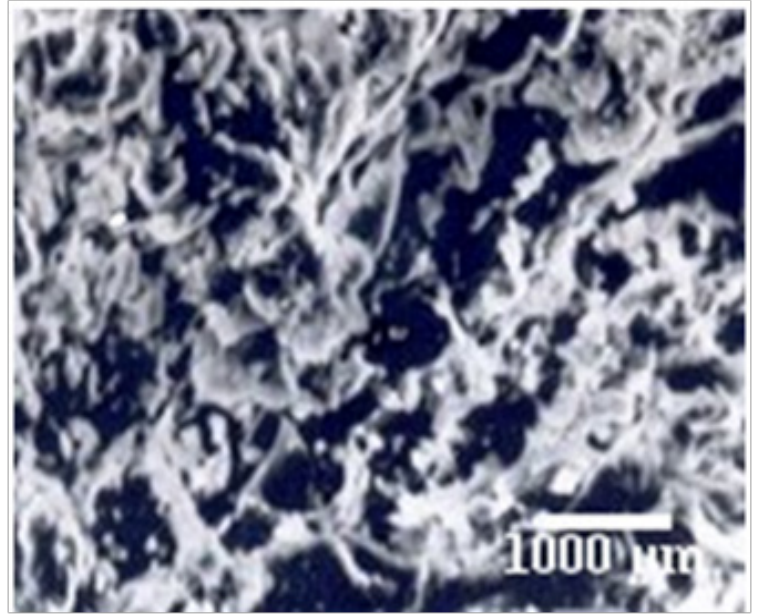

Figure 4A SEM sections of gr A depicted fibrocartilagenous tissue filled in the defect center (IOX).

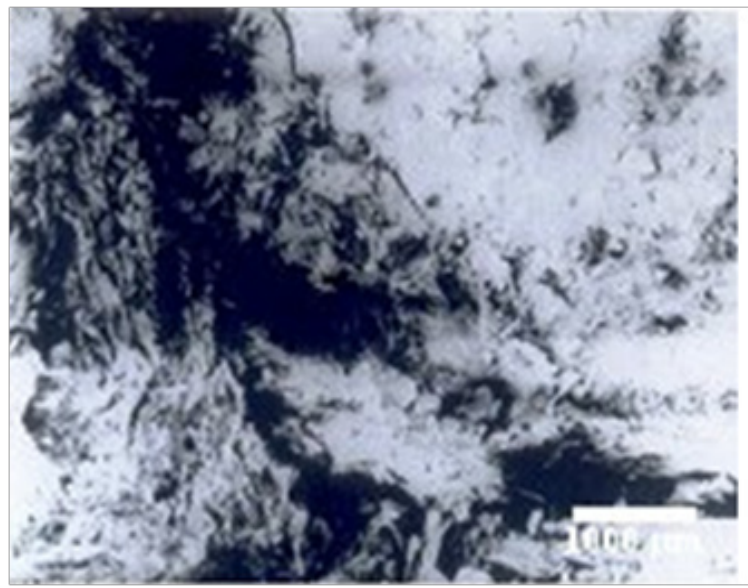

Figure 4B SEM sections of gr B showed the defect filled by ceramic particles surrounded by bony matrix but incomplete bridging between the cut ends and the implants (IOX).

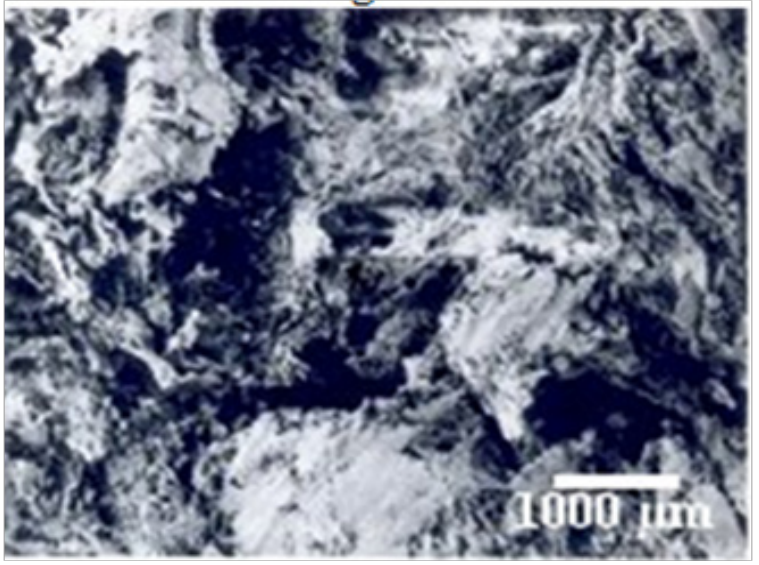

Figure 4C SEM sections of gr $C$ revealed lying down of bony matrix and fusion of bioceramic implant with newly formed bone (IOX).

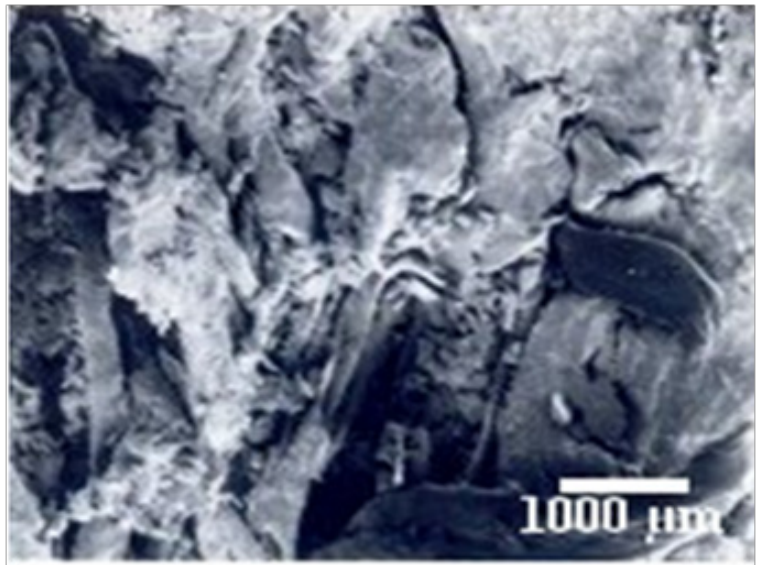

Figure 4D SEM sections of gr $D$ showed newly formed bony matrix as well as cartilaginous matrix at the defect site (IOX).

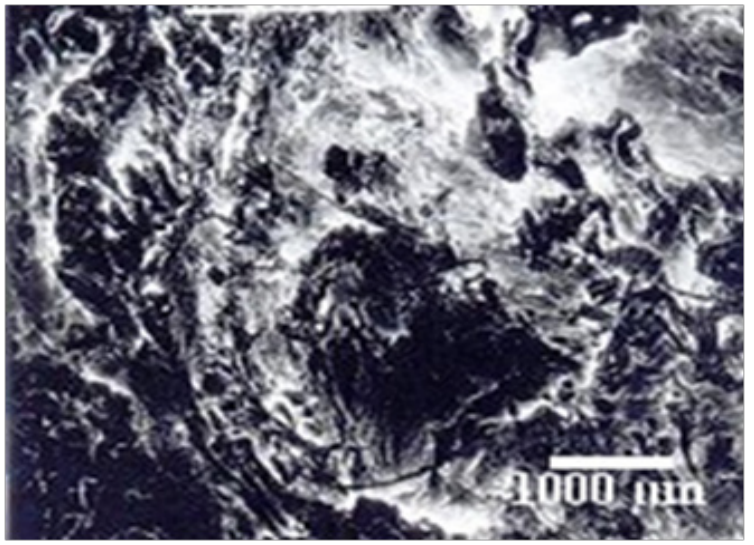

Figure 4E SEM sections of gr E depicted trabecular new bone formation evidenced by irregular areas on the surface of bone defect (IOX).

\section{Discussion}

The aim of the present work was to study the effect of ESC with growth factors (BMP-2, TGF- $\beta$ and IGF-1) added on a resorbable bioceramic scaffold (HA/TCP) in the regeneration of bone at segmental 
( $5 \mathrm{~mm})$ bone defect, using rabbit model. The experimental model used in this study was the standard procedure to create $5 \mathrm{~mm}$ defect in the radius bone of skeletally mature New Zealand White rabbits. While making the defect, the site was copiously irrigated with cold saline to prevent the thermal necrosis of the cut edges. The periosteum was also removed from the whole host bone so that the external sources of osteoprogenitor cells were absent either from the cut edges or from the host bone. The defect was filled by HA/TCP granules which were secured in position by suturing the muscle and fascia over the implant. A similar technique has been suggested by various workers for the creation of osteotomy defect and holding the graft material in situ in rabbits. ${ }^{18,19}$ The segmental bone defect was not fixed by any internal or external fixation. Since the radius and ulna have adhered together tightly with the interosseous membrane, adequate stability was achieved after osteotomy by leaving the ulna intact without any fixation of the radius.$^{20}$ In addition to this, application of a lightweight wooden splint prevented fracture of ulna as well as the migration of HA-TCP granules towards the periphery. Attempts were made to keep most of the variables constant so as to evaluate different treatment groups. The model proved to be successful for this purpose. The use of ceramics in bone and joint surgery has received considerable attention, particularly as permanent implants for joint replacement. Biologically active ceramics such as TCP, HA and ALCAP have been considered for use as biodegradable bone replacements. ${ }^{7}$ They act as osteoconductive agents and are integrated with the new osseous tissue. ${ }^{21}$ However, the major disadvantage in use of these materials is the absence of osteoinductive properties.

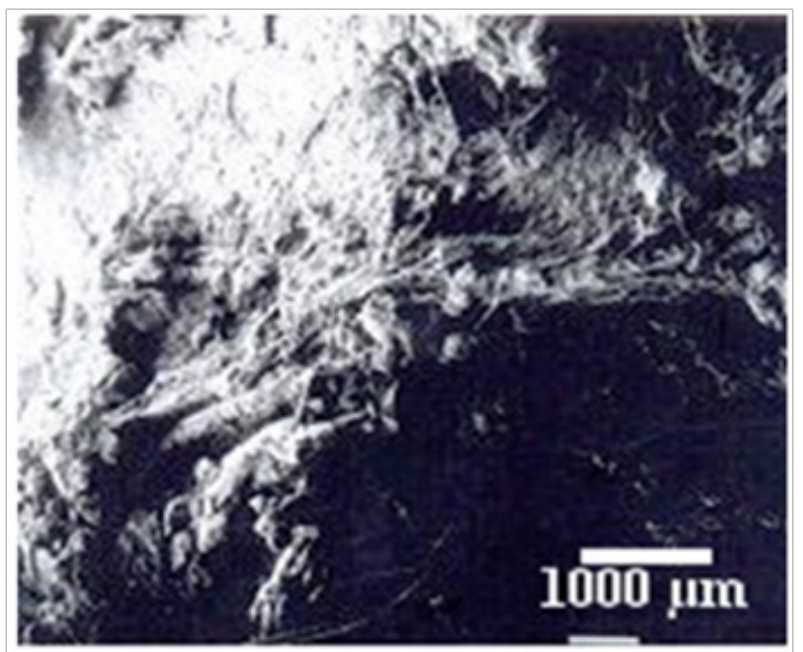

Figure 4F SEM sections of gr $F$ showed accelerated healing process at the host implant interface by the appearance of more compact bone formation within the defect site (IOX).

With the advancement in the field of cell culture technology, many workers have also reported successful culture and transplantation of osteoprogenitor cells derived from embryonic and mesenchymal stem cells at the fracture site for augmenting the healing process in different studies. ${ }^{13,14,22}$ Protocol for superovulation, embryo recovery and collection, rabbit embryonic stem cell derivation and propagation and expression of the pluripotent marker in cultured rabbit embryonic stem cells performed in this study was found easy and very useful method. Similarly, the protocol for isolation of fetal skin fibroblast cells and their subculture used in this study were also found as very useful technique. These techniques were proved to be successful for this purpose.
Mediolateral (ML) radiographs of all the animals, taken immediately after the surgery as well as at different intervals did not show marked changes in the position of HA-TCP in all groups, which filled the gap satisfactorily. These observations suggested that the suturing of soft tissues over the implant in layers was adequate to maintain the position of bioceramic granules. During the course of healing, the granules might have been entrapped in the adhesions and the tissue invading the defect site. The technique of securing bioceramic scaffold has been followed by many workers in rabbits. ${ }^{23,24}$ Postoperatively, the osteotomy defect was clearly visible at the centre of radial diaphysis in all animals and it looked relatively less radiodense than the adjacent host bone indicating the proper surgical technique used to create a model as well as for filling the site for the study.

No significant bone formation and consolidation of the defect was achieved in any of the animals in which the defect was left untreated (control group). However, at the initial stages, periosteal reaction at the fractured ends could be detected which subsequently became smooth and round, may be due to the larger fracture gap. All of the untreated defects had the radiographic appearance of nonunion. Similar reports were recorded in segmental bone defects in canines ${ }^{25}$ and in critical size bone defects in rabbit model. ${ }^{16,17}$ Since the purpose of the present study was to investigate the influence of ESC with or without growth proteins; empty bioceramic scaffolds were used as an additional control group (B). The scaffold used in the present study was HA-TCP granules-a biphasic bioceramic of calcium hydroxyapatite and tricalcium phosphate. In the bioceramic group, very little evidence of bridging at bone defect with host bone was seen at day 40 post-implantation. The periosteal reaction adjacent to the radial defect was relatively less when compared to other treatment groups. Osteogenic process was very negligible. Similar findings were observed by previous worker who implanted porous ceramic cylinders consisting of HA and TCP for repair of segmental bone defect in canines. ${ }^{25}$ The differences in resorbability on a weight basis among the different commercial HA were probably due to differences in surface area, density, size and porosity as well as procedures adopted in manufacturing of HA granules. The change in surface area however, is reported to be the greatest factor affecting the rate of resorbability of HA ceramics. ${ }^{26} \mathrm{HA}$ appeared to be osteoinductive i.e. they served as scaffolding for bony in growth. Positive results have been reported in non-weight bearing gap models in which a mixture of HA and tricalcium phosphate (TCP) particles were used..$^{27}$ Parsons et al.,${ }^{28}$ used the HA particulate in rabbit femoral canal model and observed that HA enhanced early stabilization of bone defect. Yamamoto ${ }^{29}$ documented the mean period of 4.2 months for the new bone formation around the implanted HA filled into the bony cavities after excision of benign tumors in human clinical cases. Nevertheless, with regard to the results of the present study, HA-TCP alone is not able to complete healing of $5 \mathrm{~mm}$ segmental bone defect in rabbit model. Although more new bone formation could be seen in animal treated with HA-TCP bioceramic alone compared to the empty controls, it failed to bridge the defect even at day 40 post-implantation. Similarly, reconstruction of $1.5 \mathrm{~cm}$ segmental bone defects in the rabbit ulna using porous polylactic-co-glycolic acid (PLGA) scaffold and $3.0 \mathrm{~cm}$ segmental bone defect in rabbit radius using silica coated hydroxyapatite (HASi) bioceramic failed to bridge completely even at 12 weeks post-implantation. ${ }^{17,30}$ In our view, even if this bioceramic (HA-TCP) kept for longer duration, it might fail to bridge the bone defect completely. 
In the group $\mathrm{C}$, where allogenous ESC combined with HA-TCP was transplanted into the defect area, the increased bone formation was found compared with HA-TCP alone. Radiological, SEM and histological analysis showed bony union between the scaffold and cut ends of the host bone as well as with the adjacent ulna. This observation confirms previous experiments which demonstrated the benefit of the addition of ESC on bioceramic for improved bone regeneration. The earlier new bone formation in this group might be enhanced by the osteoinductive activity of ESCs which might have differentiated into osteogenic cells in vivo. ${ }^{31}$ Stem cells have therapeutic potential in the realm of orthopaedic surgery because of their capacity to selfrenew and differentiate into various types of mature cells and tissues, including bone. ${ }^{12}$ Embryonic stem cells (ESCs) are pluripotent cells derived from the inner cell mass of pre-implantation embryos and represent embryonic precursor cells that give rise to any cell type in the embryo. Specifically, it has been shown that ES cells can differentiate into osteogenic cells under selective culture conditions. ${ }^{13}$ The osteogenic cells are capable of in vitro producing bone nodules in three-dimensional scaffolds. ${ }^{14}$ Similarly, other types of stem cells that are mesenchymal stem cells (MSCs) seeded hydroxyapatite ceramic construct also promotes infiltration of osteoprogenitor cells, and thus enhances their subsequent mineralization and bone formation during critical-size bone defect healing in long bones. ${ }^{16,17,32}$

The BMP-2 treated constructs in group D encouraged the formation of abundant bony tissue filling the bone defect, bridging both proximal and distal interfaces as well as with adjacent ulna as early as four weeks after surgery. At day 40 after surgery, radiographic, SEM and histological analysis showed newly formed bony tissue within the implantation site, well-differentiated lamellar bone surrounding the HA-TCP granules, characterized by the number of osteocytic lacunae and Haversian systems and the presence of osteoid border at the surface of new bone trabeculae. Bone development, growth, and repair predominantly occur through the process of endochondral ossification, characterized by remodeling of cartilaginous templates. Increased radiodensity in the defect site in this group is indicative of new bone formation which may be due to BMP which induces skeletal tissue development by differentiation of mesenchyme type cells and possibly also myoblast into prechondroblast and chondroblasts and by formation of organized cartilage tissue. The new cartilage developed in response to BMP has the potential of epiphyseal growth cartilage; it institutes endochondral ossification and terminates the development of lamellar bone and marrow. The results confirmed that BMP-2 accelerate osteogenesis at the defect site by the process of osteoinduction. Stem cells are known to have the ability to undergo osteogenic differentiation under suitable conditions. ${ }^{33,34}$ Their proliferation and differentiation capacity may be enhanced by exposing them to growth factors. Growth factors are protein signaling agents that act locality to stimulate formation and proliferation of osteoblasts and thereby promote bone healing. Bone morphogenetic proteins (BMPs) are hydrophobic; non-species specific glycoprotein belongs to the expanding transforming growth factor-beta super family and has a pleiotropic function that ranges from extra skeletal and skeletal organogenesis to bone generation and regeneration. It induces de novo bone formation in post-fetal life through the process of direct (intramembraneous) and endochondral ossification and their response is dose-dependent. ${ }^{10} \mathrm{BMP}-2$ has been shown to accelerate bone healing in human and animal models. ${ }^{35,36}$ BMP-2 has the highest osteoinductive potential among all the bone morphogenetic proteins. ${ }^{37}$ There are differences between species in their sensitivity to bone morphogenetic proteins, so the result can be difficult to extrapolate from one species to another. ${ }^{38} \mathrm{~A}$ minimum threshold dose of BMP-2 is necessary for a beneficial effect to occur, but a higher dose does not necessarily result in a better outcome. ${ }^{39} \mathrm{It}$ is reported that a $2 \mathrm{~cm}$ ostectomy corresponds to a critical-size defect that does not heal spontaneously without the addition of bone grafts or any bone substitute or growth proteins. ${ }^{37}$ The BMP-2 appeared to have osteoinductive properties that were quite efficient in such surgical conditions, and combining with bioceramic material (HA-TCP) might ensure early healing. Bone morphogenetic protein-2 accelerates the rate of development of the callus and cortical union after fracture of the rabbit tibia and it stimulates bone and cartilage formation so that the callus develops and matures more rapidly. ${ }^{40}$ In agreement with this study, bone morphogenetic protein-2 accelerates healing in a rabbit ulnar osteotomy model ${ }^{2}$ and rabbit radial critical size bone defect. ${ }^{17}$

Radiographic, histological and SEM analysis in group E revealed that there was comparatively better healing pattern at the defect site than the animals of group $\mathrm{C}$ and $\mathrm{D}$. Radiographic observations on day 20 revealed the signs of new bone formation from both cut ends of radius. Radiodensity was increased at the defect site and at day 40 , further progression of the new bone thereby reducing the gap at the defect site. There was complete fusion of the implant and host bone and establishment of continuity between the cut edges. The findings of this study are incongruity with the findings of previous workers who have also observed the enhanced bone formation after application of TGF $\beta-1$ in porous natural coral implants during the healing of critical size defect in Wistar rats. ${ }^{41}$ Srouji et al..$^{42}$ also found the increased amount of radiodensity while studying the tibial defect model in rats at 4 weeks observation period. The increased osteogenesis at tibial fracture healing site after repeated local application of TGF- $\beta$ has also been documented in the rat model $1 .^{43}$ Better osteogenesis in this group might be attributable to the transplantation of ESCs along with TGF- $\beta 1$. Transforming growth factor- beta 1 (TGF $\beta 1$ ), a dimeric protein, plays an important role in the normal physiological process as well as healing of the fractured bone. ${ }^{9}$ The TGF- $\beta$ s are polypeptide growth factors encoded by a family of closely-related genes that are expressed in numerous tissues and species. Bone was one of the first tissues in which locally produced molecules with TGF- $\beta$ like activity appeared to regulate normal cellular function, and the skeletal matrix probably composes the largest reservoir of TGF- $\beta$ s. Most evidence to date leads to the conclusion that the TGF- $\beta$ s have an important role in the formation of bone and this tremendous potential in therapeutic application for non-healing osseous defects and in different types of fracture healing. ${ }^{9}$ Various in vitro studies have proved the role of TGF- $\beta 1$ at every stage of bone formation. TGF- $\beta 1$ increases bone formation in vitro mainly by recruiting osteoblast progenitors and stimulating their proliferation, thus expanding the pool of committed osteoblasts, as well as by promoting the early stages of differentiation i.e. bone matrix production. ${ }^{44,45}$ The local application of TGF- $\beta 1$ to the fracture also leads to better and faster healing as evaluated by several workers in various animal studies. ${ }^{46-48}$

Radiological, histological and SEM analysis in group F revealed that there were osteochondral union and cartilaginous transition between implant and host bone at defect site as well as with the adjacent ulna. Completion of cancellous bone formation and regions of increased neo-vascularisation with moderate resorption of construct took place at day 40 post surgery. The new bone formation was more than group C. This could be because of IGF-1 is known to induce stem cells to differentiate towards the endothelial precursor germ layer, with significantly more endothelial cells at later stages ${ }^{49}$ There was a cartilaginous transition of the scaffold and new bone formation 
in group F as IGF-1 increases collagen and non-collagenous protein synthesis in vitro. ${ }^{50}$ The IGF released from bone during bone resorption promotes osteoblasts to initiate cavity refilling while IGF production by osteoclasts creates a population of osteoblasts in proportion to the volume of bone tissue resorbed. The production of IGF by stromal cells and osteoblasts predominantly regulates the process of bone repair and extent of cavity refilling. ${ }^{51}$ In agreement with this study, different workers also reported the ability of IGF to promote in vivo bone healing. ${ }^{11,52}$ Insulin-like growth factor-1 plus MSCs can improve bone healing mostly through endochondral ossification. ${ }^{53}$ Insulinlike growth factor-1 accelerated bone healing in MSCs seeded silica coated hydroxyapatite-tri-calcium phosphate (HASi) implanted in the large critical-size bone defect in the rabbit model. ${ }^{17}$ Among the three growth proteins, insulin-like growth factor (IGF-1) was shown little edge than BMP- 2 and TGF- $\beta 1$, though there was no significant differences were recorded in terms of osteogenesis in this study.

\section{Conclusion}

The findings of this study revealed that adding of allogenous rESC on bioscaffold HA-TCP could hasten the osteoconductive properties of bioceramic and can be alternative to autogenous bone graft in the treatment of delayed and non-union. Addition of growth proteins including BMP-2, TGF- $\beta$ and IGF-1 to ESC seeded HATCP bioceramic construct could accelerate bone regeneration in a segmental bone defect model controlled study.

\section{Acknowledgements}

The authors wish to thank Dr K. P. Singh, Principal Scientist, CADRAD, IVRI, Izatnagar (UP), India for his technical assistance in evaluation of histological sections and Prof. H. Varma, Sree Chitra Tirunal Institute for Medical Sciences and Technology, Trivandrum, Kerala (India) for his technical assistance for designing and preparation of HA-TCP bioceramic. The authors also wish to thank to the Director, Indian Veterinary Research Institute, Izatnagar, India and Head, Division of Surgery, Indian Veterinary Research Institute for providing necessary facilities for this study.

\section{Conflict of interest}

There are no conflicts of interest to report.

\section{References}

1. Bostrom MP, Camacho AB. Potential role of BMP fracture healing. Clin Orthop Related Res. 1998;355(Suppl):S274-S282.

2. Bouxsein ML, Turek TJ, Blake CA, et al. Recombinant human BMP-2 accelerates healing in rabbit ulnar osteotomy model. $J$ Bone and Joint Surgery. 2001;83(8):1219-1230.

3. Einhorn TA. Enhancement of fracture healing. J Bone and Joint Surg. 1995;77(6):940-956.

4. Wilkens EB, Millis LD, Daniel BG, et al. Metabolic and histologic effects of recombinant canine somatotropin on bone healing in dogs using an unstable ostectomy gap model. Am J Vet Res. 1996;57(9):1395-1400.

5. Millis LD, Wilkens EB, Daniel BG, et al. Radiographic, densitemetric and biochemical effects of recombinant canine somatotropin in an unstable ostectomy gap model of bone healing in dogs. Vet Surg. 1998;27:85-93.

6. Kalfas HI. Principles of bone healing. Neurosurgery Focus. 2001;10(4):1-4.

7. Maiti SK, Singh GR. Ceramic biomaterials in reconstructive surgery-A review. Indian J Vet Surgery. 2003;24:1-10.
8. Ninu AR, Maiti SK, Sangeetha $\mathrm{P}$, et al. In vitro osteoinduction potential of a novel silica coated hydroxyapatite bioscaffold seeded with rabbit mesenchymal stem cell. Journal of Stem Cell Research and Therapeutics. 2016;2(1):00009.

9. Maiti SK, Singh GR. Transforming growth factor-beta (TGF- $\beta$ ) in bone remodeling. Current Science.1996;71(8):613-617.

10. Maiti SK, Singh GR. Bone morphogenetic proteins (BMP)-Novel regulators of bone formation: A perspective. Indian Journal of Experimental Biology. 1998;36:237-244.

11. Schmidmaier G, Wildemann B, Bail H, et al. Local application of growth factors (Insulin like growth factor and transforming growth factor $\beta 1$ ) from a biodegradable poly (D,L-Lactide)coating of osteosynthetic implants accelerates fracture healing in rats. Bone. 2001;28(4):341-350.

12. Paul H, George QD. Therapeutic potential of embryonic stem cells. Blood reviews. 2005;19(6):321-331.

13. Beilbly RC, Boccaccini AR, Polak JM, et al. In vitro differentiation and in vivo mineralization of osteogenic cells derived from human embryonic stem cells. Tissue Eng. 2004;10(9-10):1518-1525.

14. Chaudhry GR, Yao D, Smith A, et al. Osteogenic Cells Derived From Embryonic Stem Cells Produced Bone Nodules in Three-Dimensional Scaffolds. Journal of Biomedicine and Biotechnology. 2004;4:203-210.

15. Chen X, Schmidt AH, Mahajouri S, et al. Union of a chronically infected internally stabilized segmental defect in the rat femur after debridement and application of rhBMP-2 and systemic antibiotic. J Orthop Trauma. 2007;21(10):693-700.

16. Niemeyer P, Szalay K, Luginbuhl R, et al. Transplantation of human mesenchymal stem cells in a nonautogenous setting for bone regeneration in a rabbit critical-size defect model. Acta Biomaterialia. 2010;6(3):900-908.

17. Maiti SK, Ninu AR, Sangeetha P, et al. Mesenchymal stem cellsseeded bioceramic construct for bone regeneration in large critical-size bone defect in rabbit. Journal of Stem Cells \& Regenerative Medicine. 2016;12(2):87-99.

18. Nyman R, Magnusson M, Sennerby L, et al. Membrane guided bone regeneration. Segmental radius defect studied in rabbit. Acta Orthop Scand. 1995;66(2):169-173.

19. Zhou DS, Zhao KB, Li Y, et al. Repair of segmental defects with nanohydroxyapatite/collagen/PLA composite combined with mesenchymal stem cells. J Bioactive Comp Poly. 2006;21(5):373-384.

20. Emami MJ, Oryan A, Saedinasab H, et al. The effect of bone marrow graft on bone healing: a radiological and biomechanical study. IJMS. 2002;27(2):63-66.

21. Alexander H, John RP, John LR, et al. Calcium-based ceramics and composites in bone reconstruction. CRC Critical Rev in Biocompatibility. 1987;4:43-77.

22. Maiti SK, Shiva Kumar MU, Srivastava L, et al. Isolation, proliferation and morphological characteristics of bone marrow derived mesenchymal stem cells (BM-MSC) from different animal species. Trends in Biomaterials \& Artificial Organs. 2012;27(1):29-35.

23. Bolander ME, Balian G. The use of demineralized bone matrix in the repair of segmental defects augmentation with extracted matrix proteins and comparison with autologous grafts. J Bone and Joint Surg. 1986;68(8):1264-1274

24. Gepstein R, Weiss RE, Saba K, et al. Bridging large defects in bone demineralized matrix in the form of a powder. J Bone and Joint Surg. 1987;69(7):29-31.

25. Bruder SP, Kraus KH, Goldberg VM, et al. The effect of implants loaded with autologous mesenchymal stem cells on the healing of canine 
segmental bone defects. J Bone Joint Surg. 1998;80(7):985-996.

26. Powers DL. Resorbability of commercial hydroxyapatites in lactate buffer. In: Proceedings of the 2nd Annual Scientific Session of the Academic Surgical Research. 1986. p. 13-16.

27. Ninu AR, Maiti SK, Shiva Kumar MU, et al. Isolation, proliferation, characterization and in vivo osteogenic potential of bone marrow derived mesenchymal stem cells (rBMSC) in rabbit model. Indian Journal of Experimental Biology. 2017;55(2):79-87.

28. Parsons JR, Ricci JL, Liebrecht P, et al. Enhanced stabilization of orthopaedic implants with spherical hydroxyapatite particular in biological and biochemical performance of biomaterials. In: Christel P, Meunier A, Lee A, editors. New York; 1986. 477 p.

29. Yamamoto T, Onga T, Marui T, et al. Use of hydroxyapatite to fill cavities after excision of benign tumors. $J$ Bone and Joint Surg. 2000;82(8):1117-1120.

30. Zhang X, Qi YY, Zhao TF, et al. Reconstruction of segmental bone defects in the rabbit ulna using periosteum encapsulated mesenchymal stem cells-loaded poly (lactic-co-glycolic acid) scaffolds. Chin Med J 2012;125(22):4031-36.

31. Tian XF, Heng BC, Ge Z, et al. Comparison of osteogenesis of human embryonic stem cells within 2D and 3D culture systems. Scand J Clin Lab Invest. 2008;68(1):58-67.

32. Wang X, Wang Yu, Gou W, et al. Role of mesenchymal stem cells in bone regeneration and fracture repair: a review. Int Orthop. 2013;37(12):2491-2498.

33. Tamilmahan P, Maiti SK, Sangeetha P, et al. Culture, Characterization and differention potential of rat bone marrow derived mesenchymal stem cells. Journal of Stem Cell Research and Therapeutics. 2016;1(5):00035.

34. Sangeetha P, Maiti SK, Divya Mohan, et al. Mesenchymal stem cells derived from rat bone marrow (rBM MSC): techniques for isolation, expansion and differentiation. Journal of Stem Cell Research \& Therapeutics. 2017;3(3):00101.

35. Sciadini MF, Jojnson KD. Evaluation of recombinant human bone morphogenetic protein-2 as a bone-graft substitute in a canine segmental defect model. J Orthop Res. 2000;18(2):289-302.

36. Pluhar GE, Manley PA, Heiner JP, et al. The effect of recombinant human bone morphogenetic protein-2 on femoral reconstruction with an intercalary allograft in a dog model. J Orthop Res. 2001;19(2):308-317.

37. Minier K, Toure A, Fusellier M, et al. BMP-2 delivered from a selfcross linkable $\mathrm{Ca} / \mathrm{P}$ hydrogel construct promotes bone regeneration in a critical-size segmental defect model of non-union in dogs. Vet Comp Orthop Traumatol. 2014;27(6):11-21.

38. Itoh T, Mochizuki M, Nishimura R, et al. Repair of ulnar segmental defect by recombinant human bone morphogenetic protein-2 in dogs. $J$ Vet Med Sci. 1998;60(4):451-458.
39. Boraish S, Paul O, Hawkes D, et al. Complications of recombinant human BMP-2 for treating complex tibial plateau fractures: a preliminary report. Clin Orthop Relat Res. 2009;467(12):3257-3262.

40. Bax BE, Wozney JM, Ashhurst DE. Bonemorphogenetic protein-2increases the rate of callus formation after fracture of the rabbit tibia. Calcif Tissue Int. 1999;65(1):83-89.

41. Vuola J. Natural coral and hydroxyapatite as bony substitutes. An experimental and clinical study. Academic dissertation submitted to Medical Faculty of the University of Helsinki. 2001. p. 1-67.

42. Srouji S, Blumenfeld I, Rachmiel A, et al. Bone defect repair in rat tibia by TGF-beta1 and IGF-1 released from hydrogel scaffold. Cell and Tissue Banking. 2004;5(4):223-230.

43. Nielsen HM, Andreasson TT, Leder T, et al. Local injection of TGFbeta 1increases the strength of tibial fracture in rat. Acta Orthop Scand. 1994;65:37-41.

44. Robey PG, Young MF, Flanders KC, et al. (1987) Osteoblasts synthesize and respond to transforming growth factor-type beta (TGFbeta) in vitro. J Cell Biol. 1987;105(1):457-463.

45. Janseens K, Dijke PT, Jansens S, et al. TGF- $\beta 1$ to the bone. Endocrine Rev. 2005;26(6):743-774.

46. Marcelli C, Yate AJ, Mundy GR. In vivo effects of human recombinant transforming growth factor beta on bone turnover in normal mice. J Bone and Min Res. 1990;5(10):1087-1096.

47. Lind M, Schumacker B, Soballe K, et al. Transforming growth factor- $\beta$ enhances fracture healing in rabbit tibia. Acta Orthop Scand. 1993;64(5):553-556.

48. Tanaka T, Taniguchi Y, Gotoh K, et al. Morphological study of recombinant human transforming growth factor beta 1-induced intramembranous ossification in neonatal rat parietal bone. Bone. 1993;14(2):117-123.

49. Piecewicz SM, Pandey M, Roy B, et al. Insulin-like growth factors promote vasculogenesis in embryonic stem cells. PloS ONE. 2012;7(2):e32191

50. McCarthy TL, Centrella M, Canalis E. Regulatory effects of insulin like growth factors 1 and 2 on bone collagen synthesis in rat calvarial cultures. Endocrinology. 1989;124(1):301-309.

51. Hayden JM, Mohan S, Baylink DJ. The insulin-like growth factor system and the coupling of formation to resorption. Bone. 1995;2(Suppl 1):S93-S98.

52. Blumenfeld I, Srouji S, Lanir Y, et al. Enhancement of bone healing in old rats by TGF-b and IGF-1. Exptl Gerontology. 2002;37(4):553-565.

53. Myers TJ, Yan Y, Granero-Molto F, et al. Systemically delivered insulinlike growth factor-1 enhances mesenchymal stem cell-dependant fracture healing. Growth Factors. 2012;30(4):230-241. 